\title{
The Role of Morphology of the Thumb in Anthropomorphic Grasping: A Review
}

Visakha K. Nanayakkara', Giuseppe Cotugno ${ }^{2}$, Nikolaos Vitzilaios', Demetrios Venetsanos', Thrishantha Nanayakkara ${ }^{3 *}$ and M. Necip Sahinkaya'

'Department of Mechanical and Automotive Engineering, Kingston University London, London, United Kingdom, ${ }^{2}$ Department of Informatics, King's College London, London, United Kingdom, ${ }^{3}$ Dyson School of Design Engineering, Imperial College London, London, United Kingdom

\section{OPEN ACCESS}

Edited by: Hamid Reza Karimi,

Politecnico di Milano, Italy

Reviewed by:

Yanling Wei,

Technische Universität

Berlin, Germany

Hamid Reza Karimi,

Politecnico di Milano, Italy

Hui Zhang,

Beihang University, China

*Correspondence:

Thrishantha Nanayakkara

t.nanayakkara@imperial.ac.uk

Specialty section:

This article was submitted to Mechatronics, a section of the journal Frontiers

in Mechanical Engineering

Received: 06 January 2017

Accepted: 09 June 2017

Published: 30 June 2017

Citation:

Nanayakkara VK, Cotugno G,

Vitzilaios $N$, Venetsanos $D$,

Nanayakkara T and Sahinkaya MN

(2017) The Role of Morphology of the

Thumb in Anthropomorphic

Grasping: A Review.

Front. Mech. Eng. 3:5,

doi: 10.3389/fmech.2017.00005
The unique musculoskeletal structure of the human hand brings in wider dexterous capabilities to grasp and manipulate a repertoire of objects than the non-human primates. It has been widely accepted that the orientation and the position of the thumb plays an important role in this characteristic behavior. There have been numerous attempts to develop anthropomorphic robotic hands with varying levels of success. Nevertheless, manipulation ability in those hands is to be ameliorated even though they can grasp objects successfully. An appropriate model of the thumb is important to manipulate the objects against the fingers and to maintain the stability. Modeling these complex interactions about the mechanical axes of the joints and how to incorporate these joints in robotic thumbs is a challenging task. This article presents a review of the biomechanics of the human thumb and the robotic thumb designs to identify opportunities for future anthropomorphic robotic hands.

Keywords: thumb, morphology, grasping, manipulation, musculoskeletal structure

\section{NOMENCLATURE}

\begin{tabular}{ll}
\hline A-A & Adduction-Abduction \\
ADP & ADductor Pollicis \\
APB & Abductor Pollicis Brevis \\
APL & Abductor Pollicis Longus \\
CMC & CarpoMetaCarpal \\
CNS & Central Nervous System \\
DOFs & Degrees of Freedom \\
EMG & ElectroMyoGraphic \\
EPB & Extensor Pollicis Brevis \\
EPL & Extensor Pollicis Longus \\
F-E & Flexion-Extension \\
FI & First interossei \\
FPB & Flexor Pollicis Brevis \\
FPL & Flexor Pollicis Longus \\
IP & InterPhalangeal \\
MCP & MetaCarpoPhalangeal \\
MRI & Magnetic Resonance Imaging \\
OP & Opponenes Pollicis \\
O-R & Opposition-Reposition \\
P-S & Pronation-Supination \\
ROM & Range of Motion \\
TM & TrapezioMetacarpal \\
\end{tabular}




\section{INTRODUCTION}

Human hand is a complex biomechanical structure with interconnected bones, joints, muscles, tendons, ligaments, nerves, and numerous sensors. Hand's dexterity is attributed to its around 20 number of Degrees of Freedom (DOFs) (ElKoura and Singh, 2003). Among the five digits in the hand, the thumb is the most independent (Ingram et al., 2008) and is also different in terms of kinematics, size, and strength of its muscles. Therefore, this review focuses on human biomechanics of the thumb together with work done on robotic counterparts.

Evolution of primate hand is greatly related to its interaction with the environment in food prehension strategies, available resources, and tool making (Kivell, 2015). Early human ancestors started exploring the environment using their hands once they developed bipedal locomotion 15 million years ago (Flatt, 2002). Evidence found in fossil hominins indicates that hand's unique capabilities co-evolved with its morphology (Marzke, 2013). Early tool use studies indicate that human thumb's adaptation to create forceful precision grips and ability to resist large forces in using earliest stone tools (Oldowan) is due to relatively long thumb with shorter fingers and complex intrinsic/extrinsic muscle structure (Rolian et al., 2011). Authors in Almécija et al. (2015) compare thumb-to-finger ratio among modern apes and modern humans
(Figure 1). Their analysis shows that high thumb-to-finger ratio in humans did not change since the last common ancestors of humans and chimpanzees. The hand anatomy evolved inclusively in the palm and the thumb due to its adaptation in grasping spheroids and cylinders (Young, 2003). Hence these grasps are defined as throwing grips and clubbing grips. It has been argued that the evolution of the precision grasp in addition to the power grasp enabled human to perform in-hand manipulation of objects (Pouydebat et al., 2008). Napier (1956) classifies most grasp types into the following two different groups: power grasp and precision grasp. In power grasp, the object is held between palm and the finger surfaces with primary need for force. In precision grasp, the object is held with the tips of the fingers and the thumb with less force and high precision.

Grasps are further subdivided into grasp taxonomies according to the object geometry and associated hand configurations (Cutkosky and Howe, 1990; Feix et al., 2009). In grasping objects, fingers, palm, and the thumb share forces jointly. Three such distinctive force-sharing pairs are identified as follows: (1) pad, between the pads of the fingers and the thumb, (2) palm, between the fingers and the palm, and (3) side, between the thumb and the side of the index finger (Cutkosky and Howe, 1990). The concept "virtual fingers (VF)" is put forward in Iberall (1987) to describe one or more fingers working together as a single unit to share

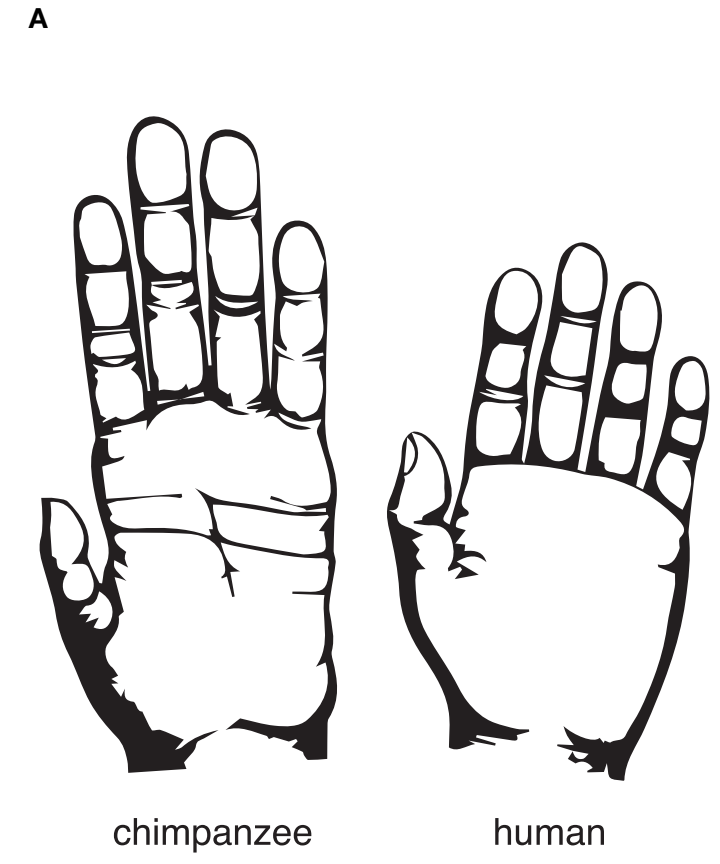

are highlighted in red and green, respectively. The figure is reprinted with kind permission from Almécija et al. (2015).

B

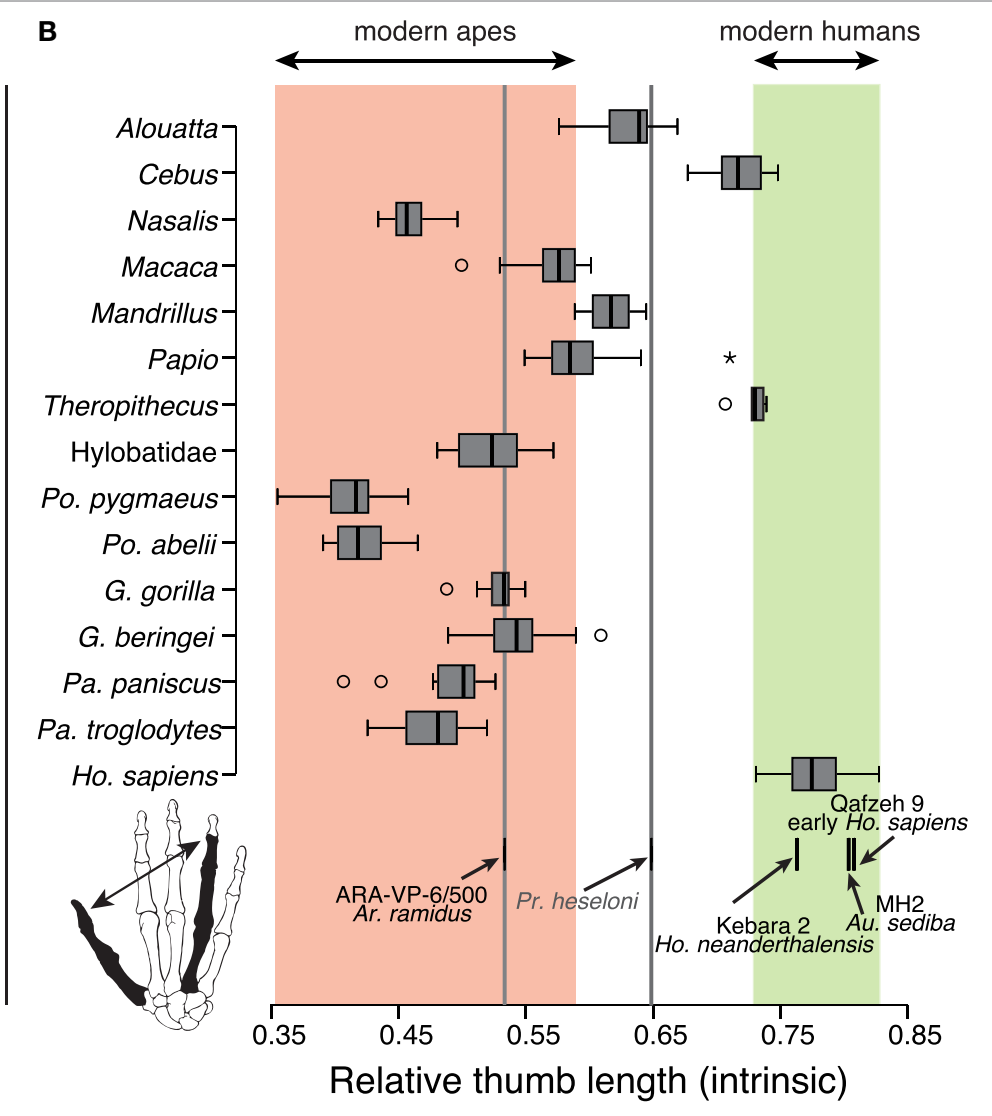

FIGURE 1 | (A) Chimpanzee and human hand comparison. (B) Thumb-to-fourth finger length ratios of modern apes and modern humans. Corresponding ranges 
grasp forces depending on the intended task. In each pair, VF1 is either palm or the thumb and VF2 consists of the four-finger combinations indicating the importance of palm and the thumb in dexterous grasping (Pons et al., 1999). Recently, existing human grasp taxonomies are analyzed and integrated into a new taxonomy called “The Grasp Taxonomy” (Feix et al., 2016). Thumb's key role in grasping objects is highlighted in this taxonomy by rearranging grasps according to thumb's Adduction-Abduction (A-A) motion. The dimensionality reduction algorithm in human and robotic hand joint space, based on coordinated movements of fingers in grasping (eigengrasps), also shows thumb's prominence in the first two eigengrasps (Ciocarlie and Allen, 2009). Human thumb along with the foldable palm contributes to make oblique arches that help to stabilize orientations and positions of the fingers in in-hand manipulation of objects (Sangole and Levin, 2008).

Early stages of robotic end effectors performed highly dexterous tasks without mimicking the human hand, for example, SARAH hand (Rubinger et al., 2001), AMADEUS hand (Lane et al., 1999). Some designs even adopt predatory bird-grasping behavior (Ramos et al., 1999). It is often argued that anthropomorphism is not a necessity in robotic hand dexterity unless the robotic hand is used in prosthesis, rehabilitation, or human-oriented purposes
(Gama Melo et al., 2014). Anthropomorphic geometry is introduced with thumb kinematics different from those of fingers in the Utah/MIT hand (Jacobsen et al., 1986) (Figure 2A). However, there are some simplifications due to practical limitations. From then onward, many robotic hands have been developed (Parida, 2013), mimicking human hand functionality (Martell and Gini, 2007). Due to their innate complex actuation strategies and cost, they are not good choices for prosthesis or wearable hands. In contrast, passively adaptive and underactuated hands are simpler in design and better for grasping in unstructured environments yet proved less dexterous (Ciocarlie and Allen, 2010).

The present prosthetic hands are not dexterous enough mainly due to the lack of functional thumbs (Carrozza et al., 2006). Adequate placement of the thumb at the right moment determines whether a grasp is successful or not (Cotugno et al., 2014). Whether the robotic thumb is properly placed in the palm can be checked by adopting some standard tests such as the Kapandji test (Grebenstein et al., 2010) and the thumb opposability index (Cerruti et al., 2015). It is important to abstract the functionality of the human thumb rather than creating a mechanical copy of its biology (Chalon et al., 2010). Thumbs in robotic hands (Grebenstein, 2012) and in exoskeletons (Heo et al., 2012) are designed based on various kinematic models. Figure 2 illustrates

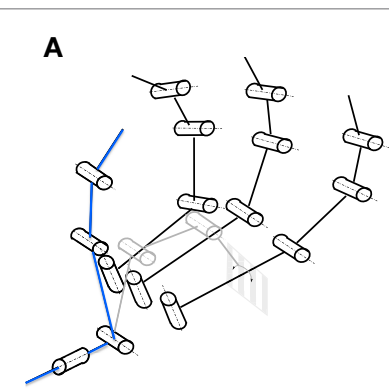

B

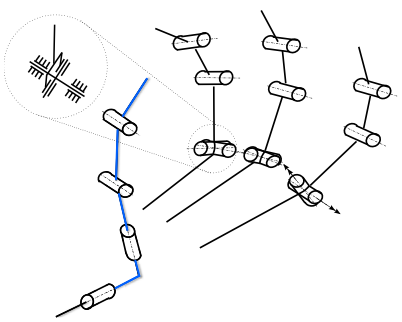

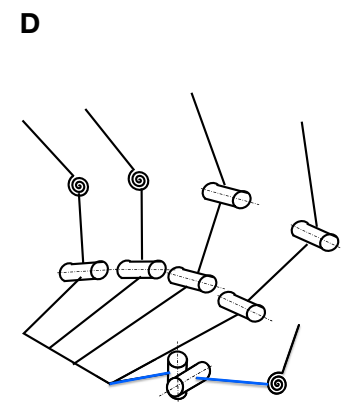

E

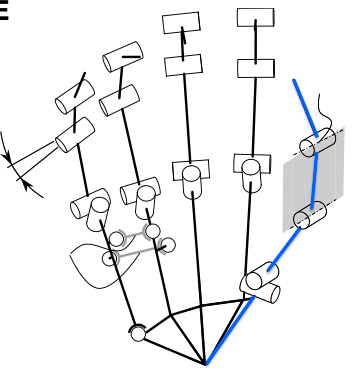

C

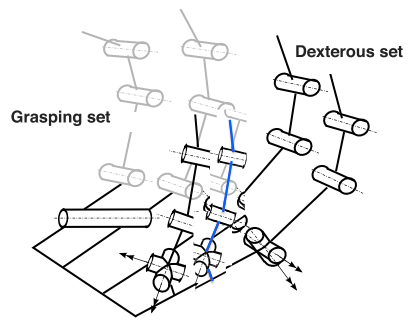

$\mathbf{F}$

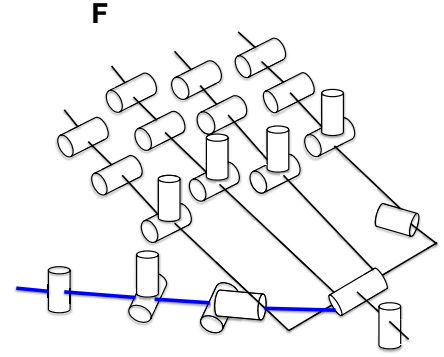

FIGURE 2 | Biomimetic thumb designs (highlighted in blue) in some selected robotic hands. The kinematic models are reprinted and highlighted with kind permission from Grebenstein (Grebenstein, 2012) and Walker (Greenhill et al., 2010). (A) Utah/MIT hand (Jacobsen et al., 1986) - two thumb proximal joint axes are separated and thumb base is placed on the palm between first and second fingers with its proximal axis parallel to the palm plane to reduce tendon routing complexity. (B) Twenty-one hand (Iwata and Sugano, 2009) - similar to the Utah/MIT hand, the two thumb proximal joint axes are separated. However, the second axis is designed to avoid thumb singulairty. (C) Robonaut hand (Lovchik and Diftler, 1999) - thumb has same finger kinematic structure and is not positioned directly opposed to the fingers. Thumb base yaw is $70^{\circ}$ and pitch is $110^{\circ}$ to increase its ROM. (D) ARMAR hand (Fukaya et al., 2000) -thumb has only 1 -DOF with its rotational axis set at an angle of $6.5^{\circ}$ to the vertical line. AA axis is positioned proximal to the palm to avoid singularity. (E) Awiwi hand (Grebenstein, 2012) -thumb has four DOFs to agree with the DLR hand arm system. Inclinations are introduced in the IP and MCP joints to improve opposition and power grasp. Thumb TM joint axes are orthogonal but not intersecting. (F) Shadow hand (Greenhill et al., 2010) -24-DOF shadow hand has a five-DOF thumb fully actuated using air muscles or smart motors. Thumb is positioned on the front of the palm with its A-A axis inclined with respect to the index finger (Grebenstein, 2012). 
biomimetic thumb designs with specific selections of thumb TrapezioMetacarpal (TM) joint axes orientations, joint locations, and passive DOFs in some selected robotic hands (Grebenstein, 2012). However, the initial kinematic model as a whole hand and mainly the interaction with the thumb has to be accurate enough in order to analyze functionality of the human hand (Grebenstein et al., 2010). These functional needs include not only grasping and manipulation but also gesturing and probing as well.

When any hand function is performed (grasp or manipulation), the real axis movement occurs not along the exact joint axes of the thumb (Griffin et al., 2000). Based on the anatomical context, the overall musculoskeletal structure plays a pivotal role in natural human thumb movement (Figures 3 and 4 ). In addition to the muscle structure, there are other supportive mechanisms including ligaments and tendons that help to maintain stability in various proportions depending on the thumb's position and forces it encounters in functional activities. Due to these additional supportive systems in the human thumb, it is necessary to make assumptions to avoid redundancies in thumb modeling. Hence, different ways of defining thumb motion (Grinyagin et al., 2005) and abstracting the thumb morphology (Giurintano et al., 1995) can be found in the literature.

This review focuses on the work done so far to understand the evolution and morphology of the human thumb and how its special biomechanical features are addressed in anthropomorphic robotic thumb designs. However, human sensorimotor contribution in grasping and intelligent robotic thumb control aspects is not within the scope of this survey. Neural bases of human hand synergies that can be extended in robotic applications are reviewed in Santello et al. (2016).

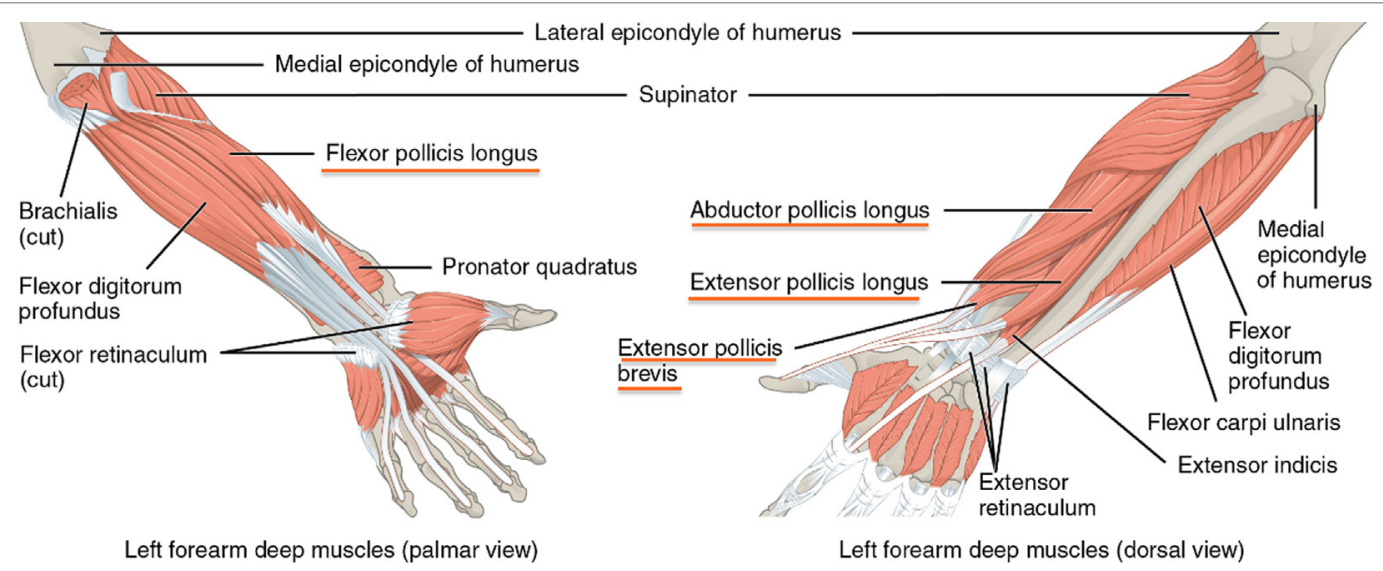

FIGURE 3 | Extrinsic muscles in the hand [https://www.lecturio.com/magazine/musculature-forearm-hand/ (Last accessed: 7/10/2016). Image credit: "Muscles That Move the Forearm" by Phil Schatz. License: CC BY 4.0 (https://creativecommons.org/licenses/by/4.0/deed.en)]. Four extrinsic muscles (see underlined) control the thumb through the tendons.

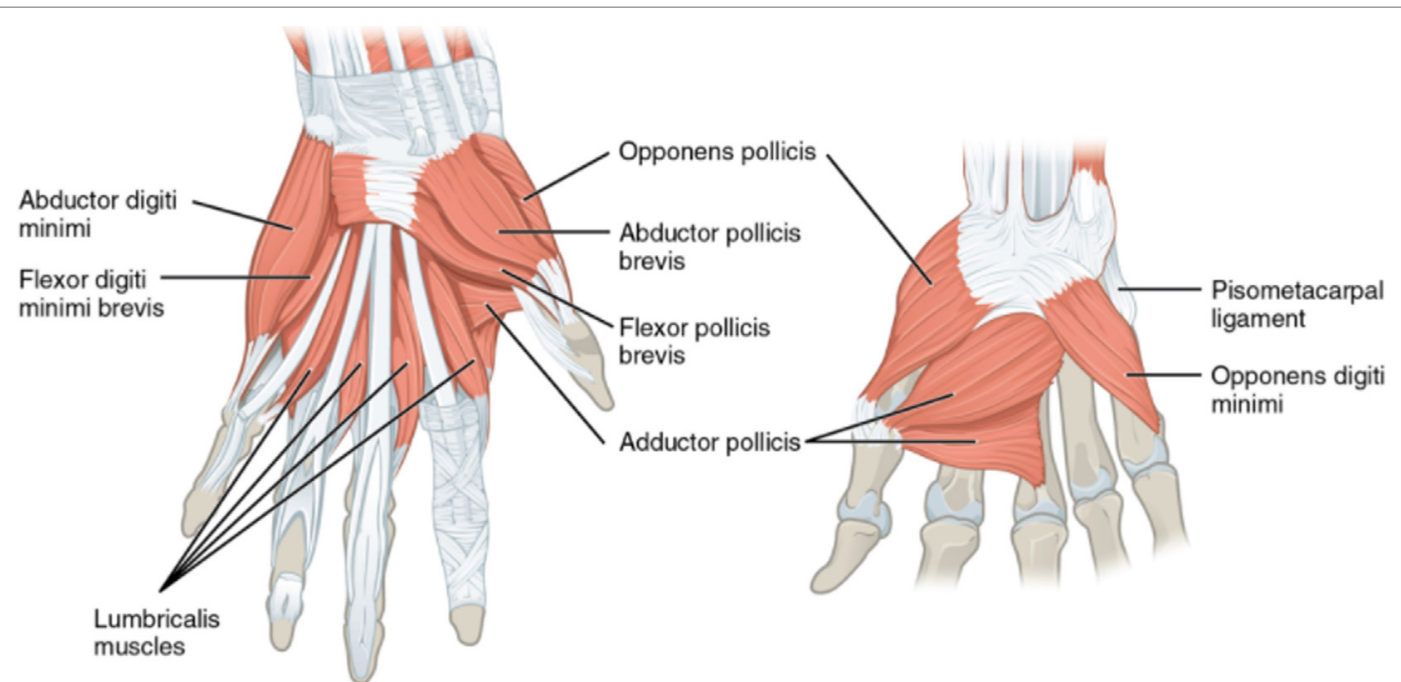

Superficial muscles of left hand (palmar)

Deep muscles of left hand: (dorsal view)

FIGURE 4 | Intrinsic muscles in the hand (http://philschatz.com/anatomy-book/resources/1121_Intrinsic_Muscles_of_the_Hand.jpg) (Last accessed: 23/06/2017). There are four dedicated intrinsic muscles to control the thumb. First interossei muscle which is attached to the adjacent sides of thumb and index finger metacarpal bones is not shown. 
In Section 2, human and non-human primate thumbs are compared in detail. Section 3 discusses thumb modeling challenges according to its natural biomechanics. Biologically inspired robotic thumbs are explored in Section 4 with their contribution in anthropomorphic grasping and stability followed by the discussion and conclusion in Sections 5 and 6, respectively.

\section{HUMAN AND NON-HUMAN PRIMATE THUMBS}

One of the most important features that granted prehistoric humans and the primates the ability to manipulate objects is the opposible thumb relative to the other fingers (Marzke, 1997; Marzke and Marzke, 2000). It can be observed that the ability of opposing the thumb to the other fingers is an infrequent skill in nature, mostly developed in humans and some primates in different ways. For example, capuchin do have dexterous manipulation skills, but their thumb is not as opposable as the human one (Pouydebat et al., 2008). Anatomical studies of the capuchin hand in Aversi-Ferreira et al. (2014) show that this observation is due to restricted or lack of medial rotation of the first metacarpal. Their adoption of a lateral pinch type of thumb opposition, which is used by humans in high precision tasks, indicates that the capuchins might use diverse neural strategies in object manipulation (Aversi-Ferreira et al., 2014). Chimpanzees, instead, have a weaker and less firm grip than humans as the muscles of their thumbs are not equally strong (Young, 2003). In contrast, the ability of gorillas to perform precision grasps more often, despite their short thumbs indicate that there could be any other factors such as musculoskeletal structure and behavioral patterns that determine grasping behaviors (Pouydebat et al., 2008).

Another difference between human and primate hands is the category of grasps that the two species prefer to use (Pouydebat et al., 2008). Adult humans prefer grasping using the fingertips (precision grasps) for small objects while distal phalanges are used for larger objects. Non-human primates prefer to use the surface of the fingers and the palm without the thumb (Pouydebat et al., 2008) even if they are morphologically similar to humans (Benefit and McCrossin, 1997). A possible hypothesis justifying the above is that our ancestor, the homo habilis, exhibited advanced skills in object manipulation in addition to object collection and tree climbing, in response to the survival needs between 2.4 and 1.4 million years ago (Haslam et al., 2009).

The authors in Rolian et al. (2011) and Marzke (2013) highlight the following key human thumb features contributed to forceful precision grasps required to make and manipulate prehistoric tools:

- Longer thumb relative to the fingers.

- Enlarged thenar muscles (the three muscles, Abductor Pollicis Brevis (APB), Flexor Pollicis Brevis (FPB), and Opponenes Pollicis (OP)) that contribute to thumb's opposition movement and hand's dexterity (Figure 4).

- Joint orientations among the trapezium, metacarpal, and capitate (Figure 5).

- Phalanx pronation due to asymmetric attachment of Flexor Pollicis Longus (FPL).
These specific human thumb features evolved over time (Lewis, 1977).

Human hand has a complex musculoskeletal structure to accomplish a complex repertoire of movements (Figures 3 and 4). There are four main groups of intrinsic muscles in the hand: thenar muscles move the thumb, hypothenar muscles move the little finger, the interosseous muscles, and the lumbricals move the other fingers. Each finger has a proximal, middle, and distal phalanx (phalanges), whereas the thumb has only a proximal and distal phalanx (Figure 5). Metacarpal bones comprise the palm. Wrist is made up from carpal bones. The joints between the carpal and metacarpal bones are called carpometacarpals. MetaCarpoPhalangeal (MCP) joints are between the metacarpals and proximal phalanges. Proximal InterPhalangeal (IP) and distal IP joints are between phalanges, whereas thumb has only one IP joint.

Human thumb has three joints: TM, MCP, and IP (since the first metacarpal of the thumb and the trapezium form the CarpoMetaCarpal (CMC) joint, it is termed separately as TM). Within the thumb, TM joint's unique saddle shape along with its unique muscle and ligament capsule contributes significantly to the stability and dexterity of any grasp (Neumann and Bielefeld, 2003). Moreover, thumb's contribution in the following three unique manipulative abilities of the human hand are identified (Kivell, 2015): the ability to rotate and in-hand manipulate objects between the thumb and the fingertips, the ability to forcefully stabilize or manipulate the grasped object between the thumb pad and one or more fingers, and the forceful grasp of cylindrical objects with the thumb either wrapping around like in a fist or stretched.

It is evaluated that the loss of the thumb corresponds to a loss of $40 \%$ of the hand functions (Hart et al., 1993). The thumb is the only digit of the hand, which can be opposed to the other four fingers, although it is composed of only two bones: the proximal and distal phalanxes. The opposition mechanism, however, involves a larger set of bones (Figure 5), namely the trapezium, the trapezoid, and the scahpoid (Kapandji, 1982). Yet there is no common agreement on how the mechanism works in detail (Emerson et al., 1996). The thumb is the only finger whose IP joint can be bent backward when the digit is fully abducted from the palm (hitchhacker's thumb) (Gray, 1918). For these reasons, the kinematic model of the thumb is one of the main sources of variability in designing kinematics of human hands, as kinematic models may vary between 15 (Bianchi et al., 2013) and 25 DOFs (Santello et al., 2013).

\section{MODELING NATURAL BIOMECHANICS AND CONSTRAINTS OF THE THUMB}

Various efforts have been made to understand the functional structure of the human hand (Taylor and Schwarz, 1955). Human hand's key parameter identification is done using Magnetic Resonance Imaging (MRI) (Stillfried et al., 2014), optical motion capture (Miyata et al., 2004), instrumented data gloves (Griffin et al., 2000), and vision-based tracking systems or using cadaver data (Giurintano et al., 1995; Valero-Cuevas et al., 2003). However, 


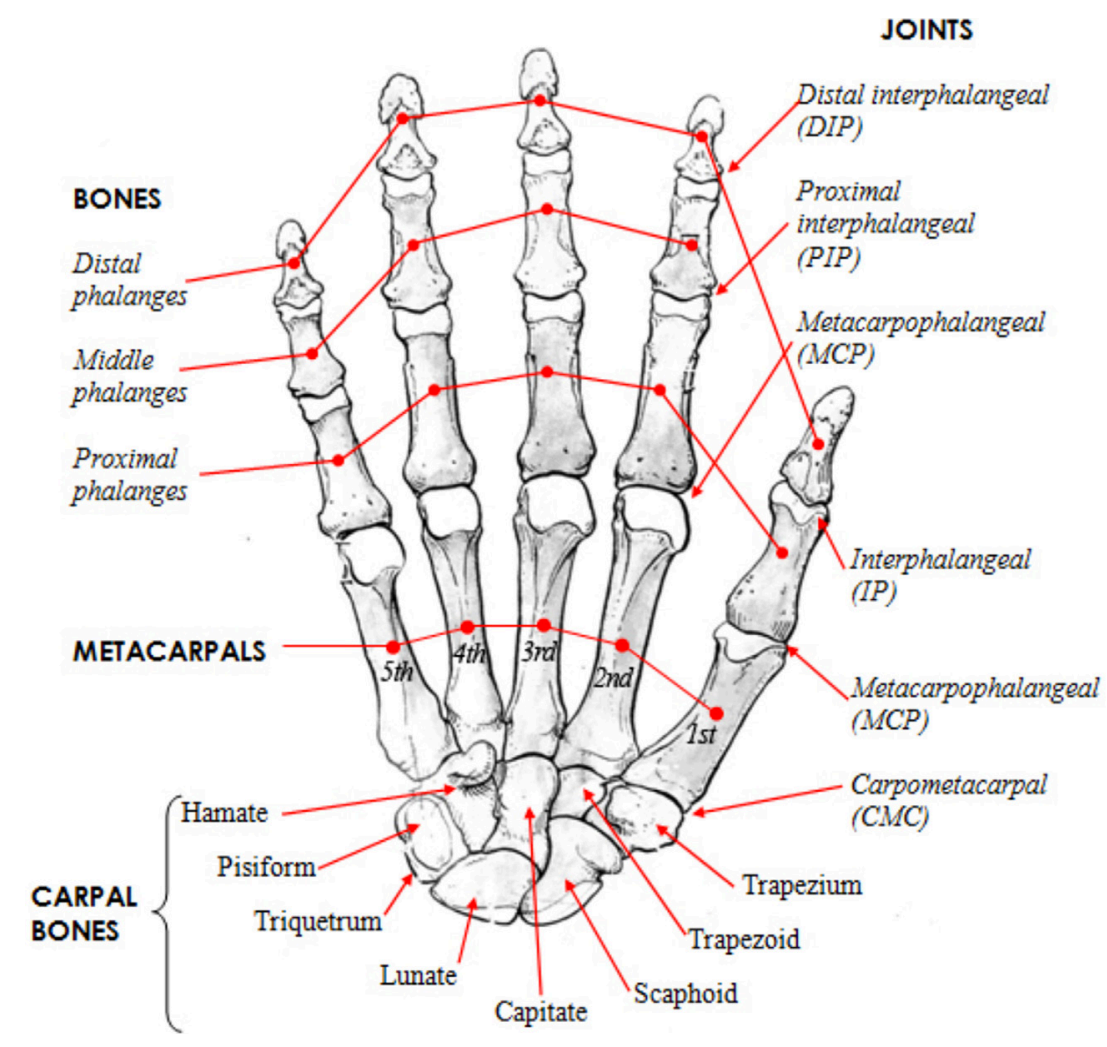

FIGURE 5 | Human hand skeletal structure depicting finger bones, joints, metacarpals, and carpal bones [http://www.amulyabharat.com/hand-bone-anatomyhuman-diagram-download/ (Last accessed: 7/10/2016)].

the high number of DOFs of the human hand in a fairly small space makes it a challenging task to record in vivo joint rotations accurately. In this context, it is important to understand musculoskeletal parameter variations (Valero-Cuevas et al., 2003) taking thumb as an embodiment in order to come up with a feasible thumb design.

The supportive role of the foldable or cupping palm, in thumb's opposition movement, cannot be avoided in the discussion of biomechanics of the thumb due to its musculoskeletal integration. Human palm's foldability and reconfigurability facilitate manipulability of the hand (Dai et al., 2009). Studies of palmar arches' contribution in hand shaping and grasping show significant involvement of the thenar arch (articular formation of the carpus, thumb MCP, index MCP, and middle MCP) (Sangole and Levin, 2008). They suggest further studies to see whether this behavior happens due to neural control or as a result of finger positions. According to van der Hulst et al. (2012), finger joints' coordinated flexion motion toward the palm causes automatic opposition and cupping of the palm against the thumb. Experimental results on the contribution of palmar arches in doing six common daily activities in Richards et al. (2015) show that the oblique arch (articular formation of thumb MCP and little finger MCP) contributes the most.

Various thumb and palm abstractions can be found in the literature. For example, human thumb joints are approximated into the following three categories depending on their particular motion in Cerruti et al. (2015): TM joint as a saddle joint (2-DOFs), $\mathrm{MCP}$ as a condyloid joint (2-DOFs), and IP joint (1-DOF) as a hinge joint. In that, the little finger CMC joint is approximated as a one-DOF hinge joint to form the palm cupping motion with the thumb. The authors in Wohlman and Murray (2013) point out that the thumb tip forces cannot be simulated accurately by individual intrinsic muscle contributions. They conclude that coordinated muscle actions (including muscle-tendon actions of the extrinsic muscles) and biomechanically defined thumb joint axes of rotation are important to be incorporated in thumb modeling for accurate thumb-tip force reproduction. However, current biomechanical thumb models do not serve this purpose. There is a lack of detailed studies on thumb musculoskeletal structure and less consistency across the available studies (Wohlman and Murray, 2013). Natural biomechanics of the human thumb are analyzed in this section to see whether there are any particular thumb musculoskeletal features that contribute to its unique motions along with an insight into the kinematic modeling of those thumb features.

\subsection{Thumb Musculoskeletal Arrangement}

\subsubsection{Thumb Joints}

Rotational axes of the three thumb joints are investigated by many researchers. There are contending findings about the TM joint's DOFs. According to Cooney et al. (1981), Pronation-Supination (P-S) motion is not independent from Flexion-Extension (F-E) 
and A-A within the TM joint. According to Hollister et al. (1995) and (Hollister et al., 1992), A-A axis of TM is in the first metacarpal, whereas F-E axis is in the trapezium. These cadaver studies show that A-A and F-E axes are not orthogonal to each other, they do not intersect, and they are not in the anatomic planes. Studies based on cadaver hands in Imaeda et al. (1994) also prove the same TM axes arrangement and record an instantaneous center of rotation movement between the trapezium and the first metacarpal base. This behavior approximates TM joint to a saddle joint. However, the irregular shape and movement (Kuczynski, 1974) of the trapezium bone at this joint hinders finding the exact location of its rotational axes (Xu and Todorov, 2016). A slight twisting of the trapezium with respect to the scaphoid and trapezoid (Figure 5) facilitates first metacarpal rotation in full opposition motion of the thumb (Neumann and Bielefeld, 2003). Thumb's opposition is governed merely not only by the joint axes but also by the ligament and muscle structure (Cooney et al., 1981). The mostly accepted thumb joint DOFs among the robotics community are as follows: TM: 2-DOFs, MCP: 2-DOFs, and IP: 1-DOF (Bullock et al., 2012).

The Range of Motions (ROMs) among the four thumb angles (rotation, abduction, and flexion at the MCP and IP joints) show distinctive differences when humans shape to grasp imaginary objects (Santello et al., 1998). Active ROM of the thumb MCP joint shows a bimodal distribution in Hume et al. (1990). Individual ROMs of the MP and IP joints are drastically different across subjects (in some cases $>300^{\circ}$ ) (Flatt, 2002). A summary of thumb joint ROMs reported based on clinical study literature is listed in Table $\mathbf{1 .}$

However, when the thumb moves as an embodiment such as in grasping, individual MCP and IP joint motions may not be effective because the TM joint mechanism predominantly controls the thumb linkage. Authors in Cooney et al. (1981) conclude that TM joint's enveloping muscle and ligament arrangement along with the MCP joint contribute to thumb's stability. According to thumb circumduction ROM studies in Zhang et al. (2005), shapes, orientations, and volumes of the two trajectory cones corresponding to thumb's two opposite direction motions are not alike. Based on these non-invasive studies, they point out that uneven muscle activation patterns and dependency of muscle passive forces on rotation direction could cause this difference.

According to Neumann and Bielefeld (2003), thumb's full opposition accounts for $45^{\circ}-60^{\circ}$ of its medial (internal) rotation. The analysis of the reachability space of the human thumb and the other fingers (Cotugno et al., 2016) shows that the thumb has a dominant role in defining a grasp configuration as the position of the other four fingers is determined by the configuration of the thumb irrespective of the object geometry.
Thumb's opposition motion results in as a combined motion of TM's flexion and adduction along with the first metacarpal pronation, while thumb's reposition results in as a combined motion of TM's extension and abduction along with the first metacarpal supination (Neumann and Bielefeld, 2003). Apart from the above reported intra-joint coordination pattern, there is inter-joint coordination across all thumb joints in flexion ( $\mathrm{Li}$ and Tang, 2007). Unique Opposition-Reposition (O-R) motion with the plane of the palm and TM joint's rotary movement causes the thumb to act in different planes in which F-E (Taylor and Schwarz, 1955) and A-A can take place, causing difficulty in assigning classic planes to thumb's movements and joint axes' orientations (Grinyagin et al., 2005).

\subsubsection{Thumb Skeletal Muscles and Tendons}

Mechanical stability and motion control of the thumb linkage depends on the relationships of the distances of enfolding muscles from joint axes of rotation (Hollister et al., 1992). Based on findings from cadaver hand studies in Smutz et al. (1998), the contribution of muscular forces and tension in ligaments on TM joint stability is pointed out in Neumann and Bielefeld (2003). A set of four extrinsic muscles control the thumb bones via tendons, whereas five intrinsic muscles are located within the palm as shown in Figures 3 and 4 . These muscles along with their specific functions are listed in Table 2. Thumb muscles in combination or in isolation move thumb joints in different directions (Greene and Roberts, 2015).

There are interconnections in this arrangement due to the existence of extrinsic muscle tendons attached to intrinsic muscles (Chalon et al., 2010). Table 2 shows that there is no extensor muscle within the intrinsic arrangement. Thumb extension is controlled by extrinsic muscles (Schieber, 1995). However, there are exceptions. APB and ADductor Pollicis (ADP) intrinsic muscles are attached to the thumb extensor mechanism (Adewuyi et al., 2016). From these two, APB is the prime mover of the thumb's opposition (Cooney et al., 1984). Specifically, it acts along with FPB, OP, and ADP to cause flexion, A-A, and rotation of the first metacarpal toward the palm until the whole thumb moves to a desired position (Colditz, 2000). This mechanism stabilizes the TM and MCP joints paving the way to transfer force distally through the extrinsic flexor, FPL, which crosses all the three thumb joints contributing to the inter-joint coordination in flexion (Ma et al., 2013).

FPB intrinsic muscle supports TM and MCP joint flexion and adduction. Inter-joint coordination between these two motions can be observed only during thumb's initial opposition stage (Li and Tang, 2007). As pointed out in McFarlane (1962), even though the intrinsic muscles are grouped according to ulnar and

TABLE 1 | Human thumb joint ROMs (in degrees) based on anatomical and experimental studies reported in literature.

\begin{tabular}{|c|c|c|c|c|c|c|c|}
\hline Thumb DOF & IP F-E & MCP F-E & MCP A-A & MCP P-S & TM F-E & TM A-A & TM P-S \\
\hline Cooney et al. (1981) & & $56 \pm 15$ & $19 \pm 8.8$ & & $52.9 \pm 11.4$ & $42.4 \pm 4$ & $17.2 \pm 9.5$ \\
\hline $\begin{array}{l}\text { Smutz et al. (1998) } \\
\text { Li and Tang (2007) }\end{array}$ & 80 & 70 & 30 & & 45 & 40 & \\
\hline Opposition & $44.1 \pm 19.7$ & $41.6 \pm 12.6$ & $12 \pm 5.1$ & $13.9 \pm 7.2$ & $50.7 \pm 7.2$ & $26.4 \pm 5.5$ & $44.7 \pm 8.8$ \\
\hline Circumduction & $35.5 \pm 18.8$ & $59.3 \pm 16.1$ & $36.8 \pm 10.3$ & $33.4 \pm 6.6$ & $63.4 \pm 9.4$ & $47.8 \pm 6.3$ & $58.4 \pm 12.3$ \\
\hline
\end{tabular}


TABLE 2 | Thumb extrinsic and intrinsic muscles (Figures $\mathbf{3}$ and $\mathbf{4}$ ) with corresponding thumb joint movement controls based on anatomical studies (F-Flexion, E-Extension, Ab-Abduction, Ad-Adduction, P-Pronation, S-Supination).

\begin{tabular}{|c|c|c|}
\hline Extrinsic thumb muscles & Functions & $\begin{array}{l}\text { Extrinsic muscle-controlled } \\
\text { joint movements (von } \\
\text { Schroeder and Botte, 2001) }\end{array}$ \\
\hline Flexor Pollicis Longus (FPL) & $\mathrm{F}$ & $\mathbb{P}(\mathrm{F})$ \\
\hline Extensor Pollicis Brevis (EPB) & mild $A b$ and $E$ & TM (E, Ab), MCP (E), IP (E) \\
\hline Abductor Pollicis Longus (APL) & $\mathrm{E}$ and $\mathrm{Ab}$ & Thumb and wrist $A b$ \\
\hline Extensor Pollicis Longus (EPL) & $\mathrm{E}$ and $\mathrm{Ad}$ & $\begin{array}{l}\text { MCP }(E), I P(E) \text {, first metacarpal } \\
\text { rotation, thumb Ad }\end{array}$ \\
\hline Intrinsic thumb muscles & Functions & $\begin{array}{l}\text { Intrinsic muscle-controlled } \\
\text { joint movements } \\
\text { (McFarlane, 1962) }\end{array}$ \\
\hline Opponenes Pollicis (OP) & $\mathrm{F}$ and $\mathrm{Ab}$ & $\begin{array}{l}\text { Thumb Ab, P, opposition, MCP } \\
\text { (F) jointly with APB and FPB }\end{array}$ \\
\hline Abductor Pollicis Brevis (APB) & $\mathrm{Ab}$ & Same as above \\
\hline Flexor Pollicis Brevis (FPB) & Ad and $F$ & Same as above \\
\hline ADductor Pollicis (ADP) & $\mathrm{F}$ and $\mathrm{Ad}$ & $\begin{array}{l}\text { Thumb S and Ad, MCP (F) } \\
\text { jointly with FI }\end{array}$ \\
\hline First Interossei (FI) & $\begin{array}{l}\text { Thumb base } \\
\text { Ad (radially) }\end{array}$ & Same as above \\
\hline
\end{tabular}

median nerve innervation (Figure 6) for anatomical analysis, in reality some muscles and hence their actions are innervated by both nerves. It is pointed out in surface ElectroMyoGraphic (EMG) control prosthetic applications (Bitzer and Van Der Smagt, 2006) that the correct positioning of the electrodes on the relevant muscles to get the EMG signals is critical to obtain a high success rate.

Since TM joint contributes to the most part of thumb's mobility, the joint's stability is maintained by its ligament formation and attachment of the tendon of Abductor Pollicis Longus (APL) (Table 2) (Lewis, 1977). Due to this intricate arrangement, most thumb muscles are multifunctional and they also act in concert. Therefore, as pointed out in Section 3.1.1, assigning classic planes to thumb motion is challenging.

When miology of the hand is considered, thumb is the only digit in which all the intrinsic muscles are located within the carpus. The muscles of the other fingers are mostly placed on the forearm and only the tendons extend to the hand to control the fingers (Gray, 1918). Additionally, the thumb is the strongest finger of the hand, along with the little finger (An et al., 1985). The human tendons are designed to position the bones or to store motion energy like a spring. The tendons are mostly composed of collagen fibers (86\%) and elastin (2\%) (Lin et al., 2004). The first type permits resistance to tensile stresses in the structure, while the second type makes the structure elastic and allows motion energy storage. Hence, the tendons can stretch and rewind to generate force when needed. The ability of the tendons to slide between and within the fascicles lets independent tension transmission irrespective of joint movements (Benjamin et al., 2008). The authors further argue that the frequent use of radial side (thumb, index, and middle finger) for power and precision grasps may account for the fact that less variation of tendons and neural connectivity is found on the radial side in contrast to the ulnar side (little and ring finger).

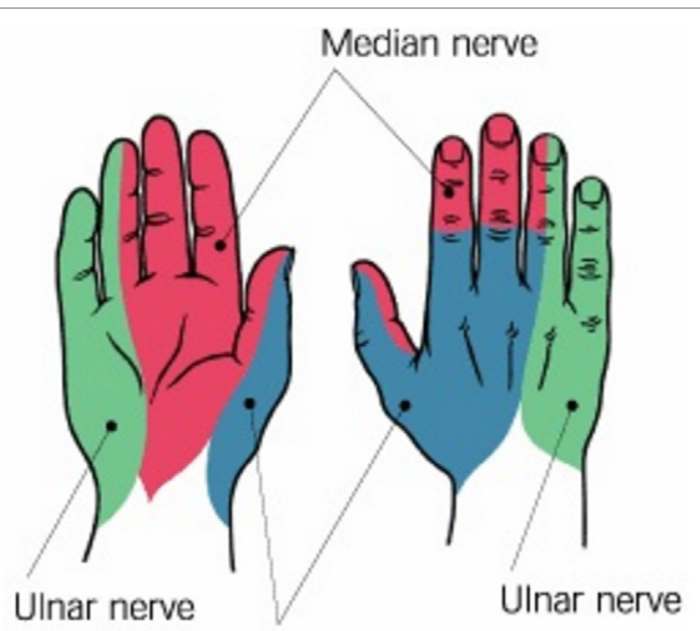

Radial nerve

FIGURE 6 | Nerves in the hand: radial, median, and ulnar (Peripheral nerves of the upper extremity. OrthopaedicsOne Clerkship. In: OrthopaedicsOne The Orthopaedic Knowledge Network. Created November 25, 2010, 14:08. Last modified November 25, 2010, 14:08 ver.2. Retrieved June 23, 2017, from http://www.orthopaedicsone.com/x/Q4DEAg).

\subsection{Thumb Neural Control}

Among the three principal nerves that serve the hand and wrist (Figure 6), radial nerve serves thumb's extensor muscles, whereas median nerve serves abductors, opponens, and flexors of the thumb (Taylor and Schwarz, 1955). Ulnar nerve is connected to the little finger and half of the ring finger. The muscles of the thumb are also controlled by alpha and gamma motor neurons, which contract a muscle spindle sending a train of impulses of variable frequency from the brainstem (Kandel et al., 2000). The control of the thumb during grasping is synergistic with the rest of the other fingers of the hand. This means that the thumb displaces together with the other digits of the hand rather than on its own (Santello et al., 1998) within the 74 and $81 \%$ of a grasping motion (Jeannerod, 1984), but the thumb can also be independently controlled. This is due to the fact that the extrinsic muscles act on thumb are isolated from the multitendoned muscle arrangement of the fingers allowing the thumb control rather independent (Kilbreath and Gandevia, 1993). Thumb tendons also have Golgi organs, which are sensory receptors that provide direct proprioceptive information on the tension of the tendons (Kandel et al., 2000).

Authors in Kilbreath and Gandevia (1993) point out the reliance of thumb muscles in grasping force control. Their findings show that neural control of muscles necessary for hand dexterity is prominent in thumb muscles rather than that of index finger muscles. They point out that this behavior might occur as a result of thumb's adaptability to various opposability forces in daily activities. Similarly, combined EMG measurements of thumb muscles and forces at the thumb IP joint in isometric contraction is done with and without median nerve (Figure 6) contribution in Kaufman et al. (1999). They analyze whether thumb muscle contributions are grouped based on innervation and confirm that FPB muscle (Table 2) is dual innervated. They 
point out that due to the existence of overlapping innervation in thumb muscles, thumb's opposition movement may be contributed by muscles innervated by the ulnar nerve (Figure 6). In contrast, Extensor Pollicis Brevis (EPB), Extensor Pollicis Longus (EPL), and APL (Table 2) extrinsic muscle tendons are innervated by the same posterior interosseous nerve (von Schroeder and Botte, 2001). Thumb joints' extensions, abductions, and first metacarpal rotation are controlled by these extrinsic muscles.

Studies in the primary motor cortex of humans that contributes to control the thumb show that out of the total area, around $85 \%$ contributes to thumb flexion with around $28 \%$ overlap with thumb extension (Zartl et al., 2014). This overlap may come from instances where the antagonistic muscles are co-contracted to stiffen the thumb. As a result, higher resolution control is done during thumb flexion (85\%) than extension and these two functions share about $28 \%$ of the motor areas.

\subsection{Thumb Kinematic Models}

Due to thumb's non-identical movement with respect to the other fingers (Battezzato, 2015) and according to grasp types (Buchholz and Armstrong, 1992), a detailed kinematic description of the thumb is a necessity. There are challenges in modeling the natural biomechanics of the thumb discussed in Section 3.1 and Section 3.2: number of DOFs and the number of associated muscles (dimensions of the model) (Valero-Cuevas et al., 2009), complex thumb joint coordination patterns (Grinyagin et al., 2005) according to the grasp and manipulation types (Santello et al., 1998), and accurate abstraction of thumb joints' axes of rotation (Wohlman and Murray, 2013). Mostly dimensionality of the model is reduced for simplicity, to avoid muscle redundancy or for the purpose being used. These issues are illustrated in some selected robotic thumb kinematic models in Figure 2. Assumptions in kinematic hand modeling including the thumb are discussed in Bullock et al. (2012).

The authors in Bullock et al. (2012) suggest that including trapezium and joint axes' location movement in thumb modeling could enhance accuracy. Thumb's dexterity increases with the number of DOFs. Even though IP joint is considered as 1-DOF in most of the kinematic thumb models (Cooney et al., 1981; Chang and Matsuoka, 2006; Chalon et al., 2010), there are competing arguments about the MCP and TM joints' DOFs (Giurintano et al., 1995; Hollister et al., 1995; Chang and Matsuoka, 2006). ROM studies based on a three-DOF TM joint kinematic model with non-intersecting, non-orthogonal A-A and F-E axes show P-S movement of $23^{\circ}$ on average (Chang and Pollard, 2008). The kinematic model developed to predict prehensile abilities of the hand in Buchholz and Armstrong (1992) has a three-DOF TM joint. However, the model can predict only the F-E angles, while the other rotation angles need to be estimated. This model is not sufficient to predict kinematics of the thumb in power grasp due to combined F-E and A-A motions at the TM joint as pointed out in Section 3.1. In contrast, MCP joint has three DOFs (A-A, F-E, and axial rotation around the thumb proximal phalanx), while TM has 2-DOFs (orthogonal and non-intersecting) and IP has a single DOF in van der Hulst et al. (2012).
A kinematic thumb model is developed in Cui et al. (2011) to match the sensor positions of the Cyberglove ${ }^{1}$ with the human thumb joints for teleoperation applications. This is done to overcome the human hand mapping problem due to kinematic model discrepancies (correspondence problem), specifically in the thumb motion. Thumb base joint location and joint axes orientation variations are analyzed using different configurations in Grebenstein et al. (2010). The authors find that TM base needs to be placed more to the front of the palm, while the intersection point of its first axis and the palm has to be distant from the finger bases. Kinematic modeling of the thumb TM and MCP joints with orthogonal and intersecting axes and fixed trapezium caused unrealistic thumb tip forces (Valero-Cuevas et al., 2003) indicating the importance of including musculoskeletal parameters/variables in the kinematic description.

As discussed in Section 3, palm plays a supportive role with the thumb in grasping. In some of the kinematic hand models, palm is included as a joint in various ways. For example, the kinematic model developed based on MRI of a single person's hand in various grasps (Stillfried and van der Smagt, 2010) includes a 3-joint palm each having 1-DOF at the bases of the four-finger metacarpals (intermetacarpals). They report each palm joint ROM as less than $23^{\circ}$, whereas thumb joint ROMs as, TM joint: $69^{\circ}$ flexion/circumduction, $48^{\circ} \mathrm{A}-\mathrm{A}$, and MCP joint: $93^{\circ} \mathrm{F}-\mathrm{E}$. Two extra DOFs are introduced at the ring and little finger metacarpal bases to include palm movement in Liarokapis et al. (2013). In contrast, the thumb kinematic model proposed in Nanayakkara et al. (2016) abstracted the foldable palm (with the thumb movement) as a virtual DOF along with variable palm parameters, which are optimized based on human grasp data. Palm parameters cannot be rigid due to the musculoskeletal motion of the thumb. Due to the significant loading on the TM joint of the thumb, merely a rigid bone structure is not sufficient to predict muscle forces during dynamic loading (Valero-Cuevas et al., 2009).

\section{BIOLOGICALLY INSPIRED ROBOTIC THUMBS}

It is a prerequisite to understand what morphological features of the human thumb discussed in Section 3.1 are most important (Jones and Lederman, 2006) and how to orchestrate this unique behavior in robotic hand designs (Bicchi, 2000) to achieve functionality, dexterity, and cosmetic appearance preferable for the robotic hands (Biagiotti et al., 2004). Successful incorporation of thumb's opposability and a desired number of DOFs enhance the preferable features of a robotic hand. However, to what extent the human biomechanical features of the thumb can be abstracted with less-sophisticated control strategies? Robotic thumb models can be found in the literature with various design and control strategies. For example, a spring-based finger and thumb mechanism is adopted in the Iowa hand (Yang et al., 2004), a twisting mechanism is introduced at the base of the thumb in Hoshino and Kawabuchi (2005) to get proper thumb tip orientation

${ }^{1}$ Virtual Technologies, Cyberglove User's Manual (1992). 
with the other finger tips, MCP joint articulation is eliminated to simplify the thumb prototype developed in Pulleyking et al. (2016) adopting fusion of the MCP and TM joints, similar to surgical intervention treatments. While summarizing some of the bio-inspired robotic thumb models in humanoid/prosthetic hands (some of the humanoid hands have prosthetic applications as well) and in exoskeletons developed so far, a brief overview is given about recently developed compliant, soft underactuated robotic hands.

\subsection{Multifingered Robotic Hand Thumbs}

Early stage multifingered robotic hands with individually articulated joints are designed to investigate machine dexterity and manipulation capability. Three-fingered Okada hand (Okada, 1982) and Stanford/JPL hand (also called Salisbury hand) (Loucks et al., 1987) thumbs are identical to the fingers except placed in opposition. Stanford/JPL hand design proves that the minimum number of DOFs to achieve manipulation dexterity in a hand with rigid, hard fingers is nine (Bicchi, 2000). Rather a anthropomorphic approach is adopted in the Utah/MIT hand (Jacobsen et al., 1986) by introducing thumb kinematics different from finger kinematics. Inaccurate joint angle control and complexity in tendon routing caused problems in these hands (Kawasaki et al., 2002). Even though embedded actuators are used to solve those problems, the number of actuators needs to be limited to keep the hand weight and size low (Butterfaß et al., 2001). In contrast, joint coupling mechanisms (rigid joint coupling) are developed to adapt the hand (passive-driven joints) to object shapes in grasping such as in Belgrade/USC hand (Bekey et al., 1990). Two motors are used in Belgrade/USC hand to rotate and flex the thumb while two other motors are used to flex each coupled finger pair.

Thumb kinematics adopted in robotic hands (Figure 2) vary based on thumb base inclination with the palm, joint axes orientations and locations, number of actuated DOFs, etc. A selected set of robotic thumb designs are discussed below.

\subsubsection{Robonaut Hand (Lovchik and Diftler, 1999), Robonaut II (R2) Hand (Diftler et al., 2011; Bridgwater et al., 2012)}

Robonaut hand has five fingers and 14 DOFs controlled by 14 motors mounted on the forearm, and power is transmitted through flex shafts. The hand can perform manipulation and grasping using two separate sections as illustrated its kinematics in Figure 2C. Only the three-DOF thumb, index, and the middle fingers do the manipulation (dexterous) set, while 1-DOF two other fingers and 1-DOF palm maintain the stable grasp (grasping set).

R2 thumb has four independently controllable DOFs. A fifth DOF is introduced in between TM and MCP F-E axes with a fixed angular twist. This design provides human-like thumb opposability. Thumb adopts the $N+1$ configuration, which is the minimum number of tendons needed for a controllable finger with $N$ number of DOFs (Inouye and Valero-Cuevas, 2013). Four out of five thumb tendons do flexion and support grasp forces against the fingers.

\subsubsection{Gifu Hand III (Mouri et al., 2002)}

Fully actuated four DOFs Gifu hand III thumb joints are controlled by built-in servomotors (Mouri et al., 2002). All the hand joints have built-in servomotors making it a compact hand with incremented complexity and high dexterity. Due to the high response of its fingers, it can exceed human hand motion (approx. $1.35 \times$ human hand speed). The hand weighs $1.4 \mathrm{~kg}$. The mobility space intersection of each finger and the thumb is used to evaluate a performance index of the thumb opposability in Mouri et al. (2002). Based on that index, Gifu hand III is 3.75 times better than that of Gifu hand II (Kawasaki et al., 2002).

\subsubsection{DLR Hand I-II (Liu et al., 1999; Butterfaß et al.,} 2001) and (DLR/HIT) Hand I-II (Liu et al., 2007, 2008)

In all DLR hand models, thumb is identical to the other four fingers to keep high degree of modularity. Each finger has 4 joints with 3-DOFs, and the distal two joints are mechanically coupled. Disadvantages due to the high integrated design of the DLR hands I-II are addressed by the modular DLR/HIT design. DLR/ HIT hand I has 4-DOFs including nine DOFs to move the thumb with respect to the palm (Liu et al., 1999). However, this extra DOF introduced for the thumb for fine manipulation and power grasping is not used as expected. Hence, in DLR/HIT hand II, the thumb is fixed at a designated angle to the palm (Liu et al., 2008). The actuators, gears, and controllers for each finger are embedded within the finger. Since DLR/HIT hand II is designed basically for dexterity, it has the lowest precision grip force to weight ratio according to robotic hand analysis in Belter et al. (2013).

Each modular finger including the thumb has two separate units for the finger base (2-DOFs) and for the finger body (1-DOF, 2 joints). Due to transmission mechanism modifications, DLR/HIT hand II shows more manipulation capability, flexible stiffness at joints, minimal tendon routing, etc., than its earlier counterparts. The size of the DLR/HIT hand II is comparable to a human hand with each finger $169.1 \mathrm{~mm}$ long (one third of the DLR/HIT hand I finger), and the weight of the hand is $1.5 \mathrm{~kg}$ (Liu et al., 2008).

\subsubsection{Anatomically Correct Testbed (ACT) Hand} (Chang and Matsuoka, 2006; Deshpande et al., 2013) ACT hand is developed to study biomechanical functions and neuromuscular control of the human hand. TM and MCP joints of the ACT thumb have non-orthogonal, non-intersecting 2-DOFs each, and IP joint has 1-DOF. Bones are exact replicates of the human bones. Separate pin joints are used to represent the TM joint F-E and A-A axes, which are located separately on first metacarpal and the trapezium bone. Narrow slot cuts in the two bones limit the joint ROM. A gimbal mechanism is used to realize MCP F-E and A-A motions, while a single pin joint is used for the IP joint. Thumb has eight actuators connected via a tendon hood structure to represent same number of muscles as the human thumb. DC motors are mounted on the forearm to actuate the tendons individually. The authors point out the importance of an extensor mechanism to further improve the tendon structure to replicate interdependencies among them (Chang and Matsuoka, 2006). 


\subsubsection{Metamorphic Hand (Dai et al., 2009;}

Wei et al., 2011)

Five finger, Metamorphic hand has a novel reconfigurable palm based on the metamorphic mechanism (Dai and Jones, 1999). The palm consists of a spherical five-bar linkage, which has two actuated joints to reconfigure it (Dai, 2005) and to move independently of the fingers (to make submechanisms). This helps to enlarge the workspace and to enhance adaptability by changing the finger positions and postures for diverse tasks. In addition, the bars can be locked allowing the hand to manipulate objects held in the palm. The thumb is fixed to one of the movable bar linkages of the palm adding another joint than the fingers making a 4-DOF thumb (Wei et al., 2011).

\subsubsection{Highly Biomimetic Anthropomorphic (HBA) Hand (Xu and Todorov, 2016)}

Authors in $\mathrm{Xu}$ and Todorov (2016) developed a highly biomimetic hand with $3 \mathrm{D}$ printed artificial bones (from laser-scanned cadaver hand bones), crocheted tendons and ligaments, laser-cut extensor hood integrated with intrinsic muscles, and an elastic pulley mechanism for the flexor mechanism. Their dimensions are decided to achieve the desired joint motions. The following three Dynamixel servo motors, out of total 10, are used to control the thumb: one for extension and abduction and two for separate flexion and adduction. Palm has a single underactuated DOF as a result of ring and little finger flexion.

\subsubsection{University of Bologna Hand, Version 3 (UBH 3)} (Lotti et al., 2004)

UBH 3 design is based on hand's internal skeletal mechanism: bones, tendons, and soft tissues. Its novel anthropomorphic behavior is obtained using rigid links, elastic joints, and flexible tendons. Due to the deformable nature of these elastic-hinged joints, the fingers are compliant. However, this mechanism shows drawbacks such as non-ideal behavior of the hinges and difficulty in modeling finger kinematics (Biagiotti et al., 2003). Thumb is similar to the fingers except placed oppose to the palm structure. Each finger has four joints. The new version, which also has A-A motion at the proximal joint uses different materials to hinges, phalanges, and tendons to address drawbacks of its earlier version (Lotti et al., 2004). The modular design facilitates either individually or combined joint actuation within a finger. Hence each finger can have up to four fully actuated DOFs including the thumb.

\subsubsection{Awiwi Hand (Grebenstein, 2012)}

The Awiwi hand is designed for both grasping and manipulation using a tendon-driven actuation system. Since the DOFs are needed to be compatible with the DLR arm system, thumb has only 4-DOFs. Thumb is actuated by four tendons attached to the metacarpal bone. Different thumb base joint positions/orientations (whether the thumb base joint is located in front of the palm or within the palm, the axes of the TM joint are orthogonal or non-orthogonal) and MCP/IP inclinations are tested prior to decide the thumb position in this hand (Grebenstein et al., 2010). Hence an inward rotation of IP joint than the MCP joint is introduced to achieve thumb's opposition motion to support key and power grasps. This inclination is optimized using the Kapandji test and extensive key and power grasp tests. A fourbar mechanism at the little finger and ring finger supports palm cupping motion. Awiwi hand's dexterity is attributed to this novel palm and thumb design.

\subsubsection{EthoHand (Konnaris et al., 2016)}

EthoHand thumb TM joint is designed as a tendon controlled ball joint, and the palm arch is obtained introducing two additional hinge joints (coupled) at little and ring finger bases to improve hand's manipulation capability. The ball joint at the base of the first metacarpal is placed on a socket at the palm. This ball-socket mechanism along with two antagonistic tendon pairs to avoid unnecessary rotation around its central axis lets the TM joint to have three DOFs. Thumb is fully actuated using the following three servos: two to control TM planar F-E and A-A motions and one to control overall thumb F-E. The proposed thumb and palm design contributed to perform four in-hand manipulation tasks (palm-arch opposition, rotate a grasped ball and a screw driver, and precise tapping on a handheld mobile).

\subsubsection{0. iCub Hand (Schmitz et al., 2010)}

Tendon actuated five-fingered iCub humanoid hand thumb has four joints. Its MCP and IP joints are coupled and are actuated by an open-ended tendon drive in which the tendon is used to flex the joints while torsional springs at MCP and IP do the extension. Hence, MCP and IP joints are compliant. Thumb TM opposition, TM A-A, index finger F-E, and middle finger F-E are actuated using a single actuator without any coupling among them. This is done using a closed loop tendon drive in which a tendon, routed through the joints and a pulley, is attached to the motor via guide wires. Hence, joint's bi-directional motion can be controlled using tendons.

In most of the robotic thumbs discussed in Section 4.1, hinges, linkages, and gimbals are used to replicate the thumb bones and joints, even though the biological ones are in sophisticated shapes and lengths and have unequal rotational axes due to musculoskeletal features as discussed in Section 3 (Xu and Todorov, 2016). For example, thumb TM joint is modeled as a three-DOF ball joint in Konnaris et al. (2016). Scanned human thumb bone shapes are first adopted in the ACT thumb mechanism (Chang and Matsuoka, 2006). Its joints are designed using pin joints and gimbals with variations to acquire biological thumb ROMs reported in literature (Table 1). The biomimetic hand design in $\mathrm{Xu}$ and Todorov (2016) adopts a detailed 3D printed MRI scanned hand bones. They point out the fact that conventional mechanical joints with fixed rotational axes do not represent human thumb movement. Salient thumb musculoskeletal features (Section 3) can be abstracted in a step by step manner ( $\mathrm{Xu}$ and Todorov, 2016). A rolling TM joint is designed using ellipsoidal magnets to replicate the conic rolling path of the human thumb first metacarpal across the trapezium in Pulleyking et al. (2016). It has been understood that a rigid palm design in most of the robotic hands limits its dexterity. Hence, reconfigurable palm (Wei et al., 2011) or extra DOFs are added to the ring and little fingers (Grebenstein, 2012; Konnaris et al., 2016) to move the palm. 
In order to achieve larger grasping force and speed, a threefingered tendon-driven robotic hand is designed in Ozawa et al. (2014) with active and passive tendons. Thumb is fully actuated with three joints driven by four active tendons. In contrast, a 15-DOF tendon-driven robotic hand uses a single actuator (Gosselin et al., 2008) to control the hand. Likewise, various actuation mechanisms are adopted to perform the muscle and tendon behavior of the thumb. Air muscles used in the Shadow hand (ShadowHand, 2013) act similar to the biological ones when actuated with compressed air. It has 40 air muscles mounted on the forearm. Human thumb's musculotendon structure as discussed in Section 3.1.2 with flexor and extensor hood is not very frequently implemented in robotic thumbs. A crocheted ligament and tendon structure is introduced in the anthropomorphic hand in $\mathrm{Xu}$ and Todorov (2016). Hybrid actuation cylinders using both air and liquid are used in Blackfingers (Folgheraiter and Gini, 2000) to produce linear movement for flexor and extensor tendons.

Compared to multifingered robotic thumbs discussed in Section 4.1, simpler thumb designs are suitable for prosthetic hands to avoid mechanical complexity by reducing the number of DOFs and hence the number of actuators with simple control. The control algorithms might mitigate the shortcomings of current thumb designs (thumb base position and orientation). However, some objects might still be difficult to grasp because the kinematic limitations have to be compensated by an adequate palm alignment (Cotugno et al., 2014).

\subsection{Thumbs in Prosthetic Hands}

Prosthetic hands are predominantly designed for grasping objects designed for the human hand and not for manipulating them (Carrozza et al., 2004). Dexterity of a hand is associated with the diversity of the tasks as well as how good it can perform the tasks (Ma and Dollar, 2011). With the current technology, it is a challenge to make prosthetic hands with dexterity and weight comparable to the human hand (an average human hand weighs about $500 \mathrm{~g}$ (Greiner, 1991)) and simple control mechanisms to actuate the large number of DOFs. In order to address these factors, the number of active DOFs needs to be reduced while compromising between the capability and the control complexity (Bicchi, 2000).

In general, thumb needs to have more than one DOF to improve the hand functionality as a whole (Light and Chappell, 2000). The user-adjustable thumb rotation movement (second DOF) along with an adaptive finger flexion mechanism increases mechanical functionality and grasp types of the childsized TBM hand (Dechev et al., 2001). The authors in Tavakoli et al. (2015) analyze actuation mechanisms of prosthetic hands (with less than 5 actuators) and highlight that the number of achievable grasps increases with an actuation of A-A of the thumb. In most of the prosthetic hands reviewed in Belter et al. (2013), thumb is actuated in F-E and along the circumduction axis (Coert et al., 2003), which is in some cases inclined by $10^{\circ}-45^{\circ}$ from the wrist axis. The authors in Belter and Dollar (2011) suggest that the circumduction axis orientation can be transformed toward or away from the little finger to join thumb
F-E and circumduction as a single DOF. This practice facilitates anthropomorphic grasping and relative timing of the grasp. Commercially available i-Limb (Troccaz and Connolly, 2008) and bebionic (Medynski and Rattray, 2011) prosthetic hands solve the problem of controlling the thumb around the circumduction axis by providing the facility for the user to manually adjust the thumb to change the grasp patterns (Belter and Dollar, 2011). This design contributes to achieve different grasp types. Further improvements are needed in prosthetic hands to enhance thumb opposition movement in order to expand grasp types (Peerdeman et al., 2014).

Current commercially available prosthetic hands (myoelectric hands) use human muscle EMG signals from only one or two electrodes to control user's hand functions. This practice is not sufficient for fine finger control or to provide sufficient finger force. Multijoint myoelectric hands need to interpret more muscle EMG signals corresponding to hand postures, to expand hand's functionality. In contrast, each joint kinematics cannot be identified from EMG data (Yang et al., 2009). Since users find difficult to interpret many simultaneous inputs from the human brain to control complicated hand kinematics, current prosthetic hands have less actuated DOFs than a human hand. Therefore, simpler synergistic approaches or simpler control designs with two-input EMG signals to close and open the fingers are adopted in practice. In contrast, most of the prosthetic hands exert high grasp forces due to non-adaptive designs. Unlike in human hands, grasp forces in these rigid hands are mainly concentrated in a small contact area (thumb, index, and middle finger contact points) (Kargov et al., 2004). Still there is room for the development of coordinated human finger movement strategies to simplify prosthetic hand control (Cipriani et al., 2008). A set of prosthetic hand thumbs are discussed here focusing on the design features.

\subsubsection{The Belgrade Prosthetic Hand} (Tomovic and Boni, 1962)

A synergistic approach is used in this pioneer prosthetic hand in which all four fingers are connected to a single cable via springs that supports fingers to wrap around the objects. All fingers are flexible with 1-DOF in each finger including the thumb. Pressuresensitive finger pads and the springs provide shape adaptability in grasping. Thumb control is delayed using a separate cable to complete the grasping task. Four-finger cable and the thumb cable are connected to a single motor axis at a pre-defined angle to provide the delay.

\subsubsection{RTR II Hand (Massa et al., 2002)}

This experimental three-fingered prosthesis hand has pulleys fitted at each axis around which the two phalanges can move freely. Wires around each pulley do flexion, while extension is done by springs. Two compression springs are connected to the two fingers in order to augment the grasp stability. When the first finger contacts the object, relative spring compresses thereby allowing the second finger to flex in reaching the object. Hence, the index finger can reach the object. A four-bar link is added to vary rotational axis of the thumb when it moves, thereby giving its A-A motion. This mechanism facilitates adaptive grasping. 
4.2.3. SPRING Hand (Carrozza et al., 2004)

SPRING hand is a 3 -finger prosthetic hand (index, middle fingers, and the thumb), which can perform adaptive grasping with relatively simple controls. An underactuated mechanism similar to the one developed in Massa et al. (2002) is successfully adapted in the SPRING hand (Carrozza et al., 2004) for intra-finger adaptation. Each finger can perform F-E motion. A three-independent-pulley differential mechanism is mounted to control the hand using one actuator while maintaining adaptability among fingers.

\subsubsection{UT Hand I (Peerdeman et al., 2014)}

UT hand I is a lock-based, tendon-pulley-driven prosthetic prototype. The joint locks used are unidirectional requiring prior configuration of desired locking direction for each joint. Thumb has three DOFs with two DC motor actuators, one for flexion and the other for opposition. Thumb is placed at an angle of $45^{\circ}$ to other fingers and the palm plane. Thumb's IP joint rotation and MCP joint rotation (an approximate ratio of 2:1 has been selected for IP:MCP joint) are coupled by a tendon, whereas flexion and opposition are not completely separated.

\subsubsection{Fluidhand III (Karlsruhe Hand)}

(Gaiser et al., 2009)

Karlsruhe hand uses flexible fluidic actuators that provide torque to the joints. When actuators are inflated under pressure, expansion forces are created causing joints to flex. Since actuators can be flexed individually, the hand can perform different grasp types. These actuators are light weight and highly compliant. Hence, the hand can perform adaptive grasping. Springs are used for finger expansion. Thumb has two actuated joints at the TM. One of them is used for thumb's opposition. MCP and IP coupled joints are passively controlled. The three-DOF base of the thumb is placed $90^{\circ}$ to the middle joint for opposition movement. The same actuation system is used in later Fluidhand versions with an advanced microhydraulic system (Kargov et al., 2007).

\subsubsection{Southampton-Remedi Hand}

(Light and Chappell, 2000)

In this 5-finger hand, 2 active DOFs are designed for thumb's separate F-E motion and combined A-A/axial rotation (circumduction). The authors point out that flexion is mostly used in grasping, and passive axial rotation with combined A-A is mostly used in stabilizing the grasp. This design allows the thumb to change its opposition plane.

\subsubsection{Cyberhand (Carrozza et al., 2006)}

Cyberhand is designed with a neural interface to employ sensorimotor controls to function the hand in order to develop natural control of a prosthetic hand rather than indirect EMG-based prosthetic (Cipriani et al., 2008). Five-fingered underactuated Cyberhand has 16 DOFs actuated by 6 motors. Two motors are dedicated for the separate control of thumb's TM A-A and F-E. Five motors to flex the five fingers are located outside the hand with a tendon wiring mechanism, while the other motor is positioned in the palm for thumb's opposition. The thumb is designed in this hand in such a way that the A-A axis is aligned with the index finger to an angle with the palm. It can perform power grasps and low-load precision grasps.

\subsubsection{MANUS-Hand (Pons et al., 2004)}

Five-fingered MANUS-hand has ten joints from which three are independently controlled using three different underactuated mechanisms. A Geneva wheel mechanism is used to move the three coupled joints of the thumb in two planes (flexed in opposition and non-opposition) using a single actuator. Hence, the hand can perform cylindrical, tip, lateral, and hook grasps. However, transmission backlash causes some noise in the thumb mechanism. Eight joints of the four fingers are directly coupled and treated as a single DOF (rigidly coupled type). Martensitic material is used to develop manually bendable joints in the fourth and fifth fingers, which help to keep the hand shape in long-term grasps.

\subsection{9. i-LIMB Hand (Troccaz and Connolly, 2008)}

Each finger of i-LIMB hand is driven by individual motors. Hand muscle contractions (myoelectric "öpen" and "close" signals) are employed to control the hand functions. i-LIMB is the only prosthetic hand that can perform more than one grasp type by locking or moving fingers by the user (Gaiser et al., 2009). Thumb can be rotated $90^{\circ}$. Hence, the user can manually rotate the thumb to adjust its position according to the grasp type.

\subsubsection{Bebionic Hand (from RSLSteeper) \\ (Medynski and Rattray, 2011)}

Bebionic hand is a myoelectric hand with preprogrammed grasp postures. Five high speed motors, located within the palm, actuate the five fingers. Thumb is manually repositioned in two ways (O-R) to achieve different grasp types, and thumb can also be aligned according to the user's needs. For example, thumb's alignment is different when it does pinch/precision grasp with the index finger than the tripod grasp with index and middle fingers. All the adjustments need to be tightened prior to electrically drive the thumb.

\subsubsection{Single Actuator Prosthetic Prototype (Belter and Dollar, 2013)}

A single actuator is used to control combined all finger F-E and thumb A-A in this prosthetic hand using a novel differential mechanism. One half of the actuator cycle is used to open/close the hand (all finger F-E), while the other half is used to bring the thumb to the desired grasping posture (thumb A-A). Thereby, four common grasp types (lateral, precision, power, and precision/power) can be done without any user adjustments.

The above thumb designs indicate the selection of thumb DOFs that are independently controlled affects the overall performance of the hand. For example, actuated thumb adduction is important to move the thumb from lateral prehension to palmar prehension (Belter et al., 2013). Since thumb flexion is often used in grasping, in Southampton-Remedi hand design (Light and Chappell, 2000), thumb flexion is an independent DOF. They also note the importance of A-A, and hence, the thumb's second DOF is combined A-A motion and axial rotation (circumduction). 


\subsection{Thumbs in Adaptive/Soft Hands}

Recent development of adaptive, soft underactuated hands provides solutions for adaptability in unstructured environments (Bonilla et al., 2014). There are easily accessible and simple approaches in designing and developing underactuated hands using rapid and adaptable fabrication techniques ( $\mathrm{Ma}$ et al., 2013). Some other design advantages of underactuation (Belter et al., 2013) are as follows: compared with rigidly linked hands, underactuated fingers can interact with the grasping object at more points and thereby more grasping force distribution occur over those points and can adapt to various grasp patterns for the same number of actuators. Rob Scharff's 3D printed robotic hand ${ }^{2}$ is inspired from human muscles and the structure of an elephant's trunk. A continuum behavior such as an octopus inspired finger mechanism $^{3}$ (Laschi et al., 2012) is obtained using a series of actuation elements in soft robotic hands (Rus and Tolley, 2015). Successful designs of these compliant fingers can be adopted in future thumb designs.

Studies are carried out to find any existing human hand muscle coordination patterns (synergies) in grasping (Santello et al., 1998) that can be developed in robotic hands (Ciocarlie et al., 2007). Muscular synergies are associated with time-varying muscle activation patterns. Based on these identified patterns, a mechanism is developed to combine human grasping synergies in intra-finger and inter-finger coordination to implement hardware synergies for a robotic hand in Brown and Asada (2007). The 17 DOFs, five-fingered robotic hand uses two DC motors due to the adoption of pulley mechanism. Thumb is not treated as a special digit in this design, and these joint couplings do not facilitate hand adaptation to the object geometry in grasping. The concept adaptive synergy used in developing Pisa/IIT Soft Hand (Catalano et al., 2014) addresses the rigidity problem. It has 18 anthropomorphic joints, one soft synergy, and a single actuator.

Postural synergies with added compliance (soft synergy) enable to understand force distribution and control in robotic hands (Gabiccini et al., 2011). The 15-DOF paradigmatic hand model adopted in their work to study postural synergies has a 4-DOF thumb to represent TM rotation, abduction, and MCP, IP F-E. Based on precision and power grasp analysis, the authors conclude that the first few postural synergies identified in Santello et al. (1998) also determine adequate grasping force. In order to implement this soft synergy model, a 15-DOF hand prototype, which has the facility to couple and actuate selected synergy patterns, is developed in Bicchi et al. (2011). Their work highlights the contribution of synergy-based models toward lesssophisticated robotic hand models. In contrast, in vivo thumb muscle functions based on EMG activities for five subjects in Kaufman et al. (1999) indicate that there are synergistic contributions in thumb muscles. These studies are useful to implement joint control strategies, which can be adapted in both prosthetic and underactuated hands alike.

${ }^{2}$ http://www.dezeen.com/2015/10/26/rob-scharff-soft-robotics-3d-printed-handresponds-to-human-grip/.

${ }^{3}$ http://www.softroboticsinc.com/.
Highly underactuated robotic hands are specifically designed to grasp a wide range of objects (Aukes et al., 2014). The design mechanisms of these hands are highly compliant while controlling many links using a single actuator. For example, the iRobotHarvard-Yale (iHY) underactuated and compliant hand designed for mobile robots is capable of doing in-hand manipulation as well (Odhner et al., 2014). Three-finger Robotiq Adaptive Gripper (Franchi and Hauser, 2014) used in industrial applications uses a separate servo motor to move two fingers around the palm, while individually controlled 3 joint fingers adapt to the shape of the objects. Four characteristic thumb mechanisms in adaptive/soft hands are elaborated below.

\subsection{1. iRobot-Harvard-Yale (iHY) Hand (Odhner et al., 2014)}

$\mathrm{iHY}$ hand is developed based on the Shape Depositioning Manufacturing (SDM) hand (Dollar and Howe, 2010), which is designed primarily to grasp objects of unknown shapes and positions. iHY hand's three underactuated and compliant fingers are designed to achieve both power and low-stiff precision grasps using a single actuator per finger. The fingers can adapt to the object shape in grasping. Coupled two fingers are positioned in opposition to the other finger, which represents the thumb. The finger pair can be rotated (A-A) in opposition to the thumb depending on the grasp type. There are five actuated DOFs to drive the tendons: three for each finger flexion, one for thumb extension at the proximal joint, and the other one for the finger pair A-A. This thumb mechanism supports independent joint range control in the proximal pin joint and distal flexor joint of the thumb.

\subsubsection{5-DOF Robotic Hand with a Single Actuator} (Gosselin et al., 2008)

The thumb has 4-DOFs, from which one DOF is for abduction. However, this DOF is not actuated but locked in a preferred position in order to bring the thumb in opposition with the index and middle finger. They use sliding pulley mechanism to achieve underactuation by distributing actuating force among the fingers using two stages of sliding pulleys, while the thumb is directly attached to a single pulley. The challenge in this mechanism is how to design the sliding pulley architecture for a proper force distribution taking into account the friction forces in the tendon system. Design issues include tendon stiffness to provide low friction (stiff kite cables) and radius of the pulleys (minimum radius of $1 \mathrm{~mm}$ ). Even though this hand performed well in enveloping grasps it failed in pinch grasps. An improved version of this thumb is proposed in Lalibert et al. (2010), in which two thumb configurations are introduced for enveloping grasp and pinch grasp. Switch between the two configurations is completed through careful tendon routing.

\subsubsection{Pisa/IIT Soft Hand (Catalano et al., 2014)}

Pisa/IIT hand adopts the adaptive synergy concept to grasp objects designed for the human hand. Hence, in order to achieve anthropomorphism, it has 19 DOFs yet controlled using a single actuator. The wrist has passive 3-DOFs and is designed in a shape to roll on one another, while palm is fixed. The hand is designed 
to withstand high impacts in unstructured environments by employing a sophisticated joint mechanism with rolling joints and elastic ligaments to replace pin joints. Since a single tendon moves all the fingers in unison, thumb movement is not treated separately. The hand can successfully grasp a wide variety of daily objects when a human operates it using a hand extension. These experimental results indicate the hand's applicability in wearable applications.

\subsubsection{Robotics and Biology Laboratory (RBO) Hand 2 (Technische Unverisitat Berlin) (Deimel and Brock, 2016)}

This soft, highly compliant, low cost hand is designed using pneumatic continuum actuators (PneuFlex) mounted on printed scaffold. The palm has two actuators with its base is designed as a circular section of $90^{\circ}$ with $78 \mathrm{~mm}$ outer and $25 \mathrm{~mm}$ inner radii. The two actuators can be inflated either together or separately. This palm behavior supports thumb's opposition movement and necessary compliance in power grasps. Since a single continuum actuator controls the thumb, its DOFs cannot be specified. Thumb's dorsal side is adopted as the contact surface against the index finger in pinch grasp to avoid mechanical complexity in using a negative thumb curvature.

\subsection{Thumbs in Exoskeletons/Hand Assistive Devices}

It is an important requirement in thumb rehabilitation to assist independent movements of the thumb. According to the survey on hand rehabilitation and assistive systems in Heo et al. (2012), a few varieties of thumb-assistive mechanisms can be found. Most of the assistive devices have not included thumb support. And in systems where the thumb is included, only F-E motion is supported, whereas opposition motion is less addressed. Since thumb's circumduction movement happens at the TM joint, any support for thumb's opposition limitations/disorders needs to be given at the TM joint (Greene and Roberts, 2015). In Ueki et al. (2012), thumb opposition is assisted adopting a mechanism to make a circular cone motion in which the tip of the cone coincides with the wrist. This rehabilitation exoskeleton thumb has four DOFs and three actuators for F-E, and opposition. The exoskeleton is developed to augment force in pinch grasps (Hasegawa et al., 2012). In this device, thumb is not driven by any actuators and the thumb extension is limited using two wires to maintain its posture. Assistance is given to the index finger, while thumb's opposition motion is supported by a ball joint at the base of the thumb. A wearable second thumb is introduced in Prattichizzo et al. (2014) as an extra-finger to enhance hand's dexterity and workspace due to its interaction with the little and ring fingers.

In recently developed hand assistive devices, underactuation designs are used along with soft, lightweight materials rather than conventional rigid link mechanisms. For example, RoboGlove (Diftler et al., 2014) is designed to provide grasp force enhancement to the human hand in strenuous and repetitive work using three linear actuators that flex all five fingers of the user hand. Since the glove supports only the grasping force rather than controlling each finger, it employs only a single tendon per finger. The soft robotic glove hand assistive device in Polygerinos et al. (2015) is designed based on molded elastometric chambers that perform finger joint movements under fluid pressure. Since thumb needs to have a twisting motion in addition to bending in opposition motion (Taylor and Schwarz, 1955), thumb actuator segments are specially designed to fulfill this requirement. Tendon-driven, polymer-based wearable robotic hand (Exo-glove Poly) is designed to support grasping for the thumb, index, and middle fingers (Kang et al., 2016). Using only two actuators, the hand can support thumb F-E and combined index and middle finger F-E.

The finger-thumb exoskeleton (HX) in Cempini et al. (2015) adopts a serial structure that provides IP, MCP, and TM F-E along with thumb rotation at the TM joint due to its articulated parallelogram mechanism. Thumb opposition movement assistance is focused on the pediatric robotic thumb exoskeleton (isolated orthosis for thumb actuation-IOTA) developed in Aubin et al. (2013). 2-DOF IOTA thumb support device TM and MCP joints are actuated using flexible cables to facilitate opposition grasp. It is designed specifically for children aged 7-12 years. Two servos and cable transmission are used to actuate the thumb.

\section{DISCUSSION}

When it comes to maintain stability, strength, and dexterity in grasping, thumb's intrinsic/extrinsic muscles and the tendon structure play a pivotal role rather than the joint geometry (Light and Chappell, 2000; Benjamin et al., 2008). The knowledge of the key features, such as biomechanical constraints (thumb axes orientations and thumb joint ROMs), muscle functions, existing joint and muscle coordinations (Santello et al., 2013), and thumb's integrity with the palm, are important to design advanced robotic thumbs. There are limitations and uncertainties in correlating and applying the findings from thumb axes orientations and ROM studies in thumb modeling (Dermitzakis et al., 2013). In fact, the findings based on thumb-index finger grasping analysis (Tagliabue et al., 2015) reveal that kinematic synergies (coupling behavior of angular joint movements) may results from muscle synergies (tendency of spatial and temporal correlation among hand muscle EMGs). The authors in Santello et al. (2013) confirm that a dimensionality reduction occurs from the mechanical DOFs in hand grasp and manipulation tasks due to the existing kinematic synergies.

Ability of the human hand to hollow the palm contributes to its versatility. It can be noted that robotic hand's manipulation capability increases with the introduction of palm (cupping or passive) movement as a DOF along with biomimetic thumb TM joints (Konnaris et al., 2016; Xu and Todorov, 2016). Findings in Shimizu et al. (1997) show that the force distribution patterns on thumb and the palm are significant in daily hand grasps. However, Table 3 shows that only a few robotic hand designs include the palm as an element in grasping and palm is not considered as a DOF in prosthetic hands. Moreover, 1-DOF robotic thumbs are positioned at a predetermined angle with the palm in most of the prosthetic hands (Table 3). Such handcrafted methods and numerical methods (Cerruti et al., 2015) are adopted to decide the thumb position in robotic hands. Analogously, transformation algorithms are developed in remote control applications to 
TABLE 3 | Robotic thumb comparison in multifingered hands.

\begin{tabular}{|c|c|c|c|}
\hline Robotic hands & $\begin{array}{l}\text { No. of } \\
\text { fingers }\end{array}$ & $\begin{array}{l}\text { Thumb and palm joint movements } \\
\text { (corresponding thumb joint ROMs) }\end{array}$ & $\begin{array}{l}\text { Palm } \\
\text { activation }\end{array}$ \\
\hline \multicolumn{4}{|l|}{ Multifingered robotic hands } \\
\hline Stanford-JPL hand (Loucks et al., 1987) & 3 & All 3 thumb joints $F-E\left( \pm 90^{\circ}, \pm 90^{\circ}, \pm 135^{\circ}\right.$ distal $)$ & No \\
\hline Utah/MIT hand (Jacobsen et al., 1986) & 4 & All 3 thumb joints F-E, TM rotation & No \\
\hline Robonaut hand (Lovchik and Diftler, 1999) & 5 & $\begin{array}{l}\text { TM (F-E, A-A), MCP-IP coupled (F-E), little and ring finger cupping (palm DOF) } \\
\text { (Grebenstein et al., 2010) }\end{array}$ & Yes \\
\hline $\begin{array}{l}\text { Robonaut II (R2) hand (Diftler et al., 2011; } \\
\text { Bridgwater et al., 2012) }\end{array}$ & 5 & $\operatorname{TM}\left(\mathrm{A}-\mathrm{A}\left(0^{\circ} \sim 74^{\circ}\right), \mathrm{F}-\mathrm{E}\left(0^{\circ} \sim 85^{\circ}\right)\right), \mathrm{MCP}\left(\mathrm{F}-\mathrm{E}\left(0^{\circ} \sim 90^{\circ}\right)\right)$, IP $\left(\mathrm{F}-\mathrm{E}\left(-10^{\circ} \sim 70^{\circ}\right)\right)$ & No \\
\hline Gifu hand III (Mouri et al., 2002) & 5 & $\operatorname{TM}\left(\mathrm{A}-\mathrm{A}\left( \pm 28^{\circ}\right), \mathrm{F}-\mathrm{E}\right), \mathrm{MCP}(\mathrm{F}-\mathrm{E}), \mathrm{IP}(\mathrm{F}-\mathrm{E})\left(\mathrm{F}-\mathrm{E}:-10^{\circ} \sim 90^{\circ}\right)$ & No \\
\hline DLR hand I-II (Liu et al., 1999; Butterfaß et al., 2001) & 4 & TM, MCP, and IP (F-E) & No \\
\hline DLR/HIT hand I (Liu et al., 2007) & 4 & TM (F-E, A-A), MCP, IP (F-E) & No \\
\hline DLR/HIT hand II (Liu et al., 2008) & 5 & TM (A-A, curling/extension), MCP, IP (F-E) & No \\
\hline $\begin{array}{l}\text { ACT hand (Chang and Matsuoka, 2006; } \\
\text { Deshpande et al., 2013) }\end{array}$ & 5 & $\operatorname{TM}\left((\mathrm{A}-\mathrm{A}) 40^{\circ}, \mathrm{F}-\mathrm{E}\left(40^{\circ}\right)\right), \mathrm{MCP}\left(\mathrm{A}-\mathrm{A}\left(15^{\circ}\right), \mathrm{F}-\mathrm{E}\left(60^{\circ}\right)\right)$, IP $\left(\mathrm{F}-\mathrm{E}\left(20^{\circ} \sim 80^{\circ}\right)\right)$ & No \\
\hline Shadow hand (ShadowHand, 2013) & 5 & $\operatorname{TM}\left(\mathrm{A}-\mathrm{A}, \mathrm{F}-\mathrm{E}\left( \pm 60^{\circ}, 0^{\circ} \sim 80^{\circ}\right)\right), \mathrm{MCP}\left(\mathrm{A}-\mathrm{A}, \mathrm{F}-\mathrm{E}\left( \pm 15^{\circ}, \pm 40^{\circ}\right)\right), \mathrm{IP}(\mathrm{F}-\mathrm{E})\left(-20^{\circ} \sim 90^{\circ}\right)$ & No \\
\hline TUAT/Karlsruhe (ARMAR) hand (Fukaya et al., 2000) & 5 & Combined 3 joints F-E & No \\
\hline Ultralight hand (Schulz et al., 2001) & 5 & TM, MCP, IP (F-E) & No \\
\hline UBH 3 (Lotti et al., 2004) & 5 & TM (F-E, A-A), MCP-IP (F-E) coupled & No \\
\hline HBA hand (Xu and Todorov, 2016) & 5 & Combined extension/abduction, flexion, adduction, palm (passive) & Yes \\
\hline EthoHand (Konnaris et al., 2016) & 5 & $\begin{array}{l}\text { TM }\left(A-A\left( \pm 65^{\circ}\right), F-E\right), M C P-I P \text { coupled } F-E\left(0^{\circ}-90^{\circ}\right) \text {, coupled ring and little finger } \\
\text { bases (palm) }\end{array}$ & Yes \\
\hline iCub hand (Schmitz et al., 2010) & 5 & TM opposition and A-A (tendon driven), coupled MCP-IP F-E & No \\
\hline Metamorphic hand (Dai et al., 2009; Wei et al., 2011) & 5 & Reconfigurable palm & Yes \\
\hline Awiwi hand (Grebenstein, 2012) & 5 & $\begin{array}{l}\text { four-bar-mechanism TM (A-A, F-E), MCP, IP (F-E), at the little and ring fingers to } \\
\text { integrate the palm }\end{array}$ & Yes \\
\hline
\end{tabular}

\section{Prosthetic hands}

The Belgrade prosthetic hand (Tomovic and Boni, 1962)

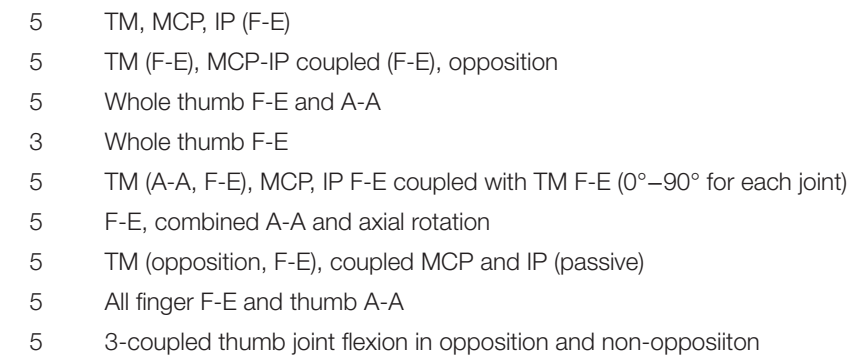

No

No

No

No

No

No

No

No address robotic and human hand dissimilarities, specifically in the thumb motion (Pao and Speeter, 1989).

Prosthetic hands provide an excellent paradigm to test alternative designs of the thumb and control systems. Since most of the current prosthetic hands use neural control approaches, users prefer to have simple kinematic designs for their long-term use. Therefore, underactuated designs are used in prosthetic hands to adapt to the shape of most frequently used objects without using complex control strategies. Adaptive synergies are successfully implemented to develop underactuated hands (Catalano et al., 2014). Yet some argue that the actuation methods and design mechanisms of prosthetic hands need to be compatible with biomechanical information from the natural hand (Carrozza et al., 2006). When robotic hands perform tasks in unstructured environments, it is unrealistic to control each and every design variable. Hence, it is useful to identify optimum design strategies to grasp objects designed for the human hand and use them to perform most frequent daily activities (Bullock et al., 2013). It can be noticed from Table 2 that the human thumb DOFs are controlled by muscles shared across different DOFs. However, most robotic thumbs from Tables $\mathbf{3}$ and $\mathbf{4}$ adopt independent joint DOFs control. Additional thumb functions can be included in a robotic hand with added complexity and weight (Belter and Dollar, 2013). Authors in Dermitzakis et al. (2013) point out that up to $76 \%$ of the work space related to the maximum ROM of the human thumb is not used to fulfill simple activities. Hence, the joint ROM and the actuated DOFs can be reduced in prosthetic thumbs without affecting the overall work space.

It should be noted that the interplay between the physical structures of the thumb and the control mechanisms plays a vital role in rendering its dexterity, though some authors emphasize the importance of one over the other. For instance, authors in Iberall (1987) point out that human hand's dexterity does not come mostly from the hand formation but rather from the 
TABLE 4 | Robotic thumb comparison in adaptive hands and exoskeletons.

\begin{tabular}{|c|c|c|c|}
\hline Robotic hands & $\begin{array}{l}\text { No. of } \\
\text { fingers }\end{array}$ & $\begin{array}{l}\text { Thumb and palm joint movements } \\
\text { (corresponding thumb joint ROMs) }\end{array}$ & $\begin{array}{l}\text { Palm } \\
\text { activation }\end{array}$ \\
\hline \multicolumn{4}{|l|}{ Adaptive/soft hands } \\
\hline 15-DOF single actuator hand (Gosselin et al., 2008) & 5 & TM A-A (locked, not actuated), thumb F-E as a whole & No \\
\hline Pisa/IIT SoftHand (Catalano et al., 2014) & 5 & Adaptive synergy actuation of all thumb joints & No \\
\hline iHY hand (Odhner et al., 2014) & 3 & F-E (independent) & No \\
\hline SDM hand (Dollar and Howe, 2010) & 3 & F-E (independent) & No \\
\hline RBO Hand 2 (Deimel and Brock, 2016) & 5 & Palm motion to support thumb opposition & Yes \\
\hline \multicolumn{4}{|l|}{ Exoskeletons/hand assistive devices } \\
\hline Device with thumb opposition assistance (Ueki et al., 2012) & 5 & $\operatorname{TM}\left(\mathrm{A}-\mathrm{A}\left(0^{\circ} \sim 60^{\circ}\right), \mathrm{F}-\mathrm{E}\left(0^{\circ} \sim 90^{\circ}\right)\right), \mathrm{MCP}\left(\mathrm{F}-\mathrm{E} 0^{\circ} \sim 60^{\circ}\right), \mathrm{IP}\left(\mathrm{F}-\mathrm{E} 0^{\circ} \sim 80^{\circ}\right)$ & No \\
\hline Tendon-driven wearable assistive device (Hasegawa et al., 2012) & 5 & TM F-E (limited E), Ad, opposition and IP F-E (limited E) & No \\
\hline RoboGlove (Diftler et al., 2014) & 5 & Combined TM, MCP, and IP joints movement & No \\
\hline Soft hand assistive system (Polygerinos et al., 2015) & 5 & MCP, IP bend (sum is $\sim 160^{\circ}$ ) and $\mathrm{E}, \mathrm{TM}$ rotation (combined $\mathrm{F}$ and $\mathrm{Ab}$ ) & No \\
\hline Exo-Glove Poly (Kang et al., 2016) & 3 & Thumb F-E & No \\
\hline Finger-thumb HX (Cempini et al., 2015) & 2 & MCP F-E, TM F-E, A-A and combined opposition & No \\
\hline IOTA thumb exoskeleton (Aubin et al., 2013) & 1 & TM F-E (ROM 65), MCP F-E (ROM 65) & No \\
\hline
\end{tabular}

Central Nervous System's (CNS) ability to fragment them into submechanisms. The same hypothesis is put forward in Tagliabue et al. (2015) saying that the CNS may not directly control joint movements (kinematics) or torques (kinetics) but their associated synergies. In contrast, studies in morphological computation show that the physical embodiment (material and musculoskeletal structure) in biological systems simplifies the computational burden on the neural controllers by solving parts of the motor control computations needed to interact with the environment (Hoffmann and Pfeifer, 2012; Pfeifer et al., 2014).

In this review, we do not cover the role of the thumb in the domain of haptic and tactile perception. It is evident that the range of manipulation capabilities also determines the range of embodied perception that is directly related to how neural representation of grasp control is organized. Recent findings in the role of internal impedance in embodied perception (Sornkarn et al., 2014, 2016), neural bases of human hand synergies (Santello et al., 2016) suggest that this is an important and interesting area to investigate in the future, because it can reveal secrets of the co-evolution of the human hand, in particular the thumb, and neural capabilities of humans. This view is also supported by the findings in Hoffmann and Pfeifer (2012) where the authors explain the link in low-level sensorymotor processes due to environmental interactions and cognition.

\section{CONCLUSION}

This review spans biomechanics of human and non-human primate thumbs, full and underactuated robotic thumbs, and robotic mechanisms covering both hard and soft hardware implementations. The gaps between the human thumb and its robotic counterparts could be found in the kinematic DOFs, mechanisms, actuators, and control schemes, given different classes of object

\section{REFERENCES}

Adewuyi, A. A., Hargrove, L. J., and Kuiken, T. A. (2016). An analysis of intrinsic and extrinsic hand muscle EMG for improved pattern recognition control. IEEE Trans. Neural Syst. Rehabil. Eng. 24, 485-494. doi:10.1109/ TNSRE.2015.2424371 manipulation tasks. As discussed in this review, the thumb has a sophisticated biomechanical arrangement and an underlying dimensionality reduction mechanism during motion control due to the existence of inter-finger and intra-thumb muscular synergies. Related to this observation, there are a few aspects that are useful in anthropomorphic robotic thumb designs. (1) There are challenges in designing mechanical joints, ligaments, and human-like flexor and extensor mechanisms to fulfill thumb's inherent DOFs and ROMs. Hence, simplifying approximations are made to model anatomical joints, muscles, and tendons. The extent of approximations in thumb modeling influences the functionality of the hand. (2) Since independent control of thumb joint motions affects the overall performance of the hand, synergistic approaches can provide viable solutions to simplify thumb models and to expand executable grasp types. Adopting muscular synergies seems to be promising to implement in robotic thumb counterparts. This is conspicuous in prosthetic thumb designs. (3) In general, the palm is not incorporated in most thumb designs, even though the thumb base musculoskeletal arrangement is integrated with it. Rigid palms in robotic hands can limit its dexterity.

\section{AUTHOR CONTRIBUTIONS}

All the authors made significant intellectual contribution in interpreting and revising the work and approved for publication.

\section{FUNDING}

This work was partly supported by the UK Engineering and Physical Sciences Research Council grants EP/N03211X/1, EP/ N03211X/2, EP/I028773/1, and EP/I028765/1 and a PhD studentship from Kingston University London.

Almécija, S., Smaers, J. B., and Jungers, W. L. (2015). The evolution of human and ape hand proportions. Nat. Commun. 6, 7717. doi:10.1038/ncomms8717

An, K., Chao, E., Cooney, W., and Linscheid, R. (1985). Forces in the normal and abnormal hand. J. Orthop. Res. 3, 202-211. doi:10.1002/jor.1100030210

Aubin, P. M., Sallum, H., Walsh, C., Stirling, L., and Correia, A. (2013). “A pediatric robotic thumb exoskeleton for at-home rehabilitation: the isolated orthosis for 
thumb actuation (IOTA)," in IEEE International Conference on Rehabilitation Robotics (ICORR), Seattle, WA, 1-6.

Aukes, D. M., Heyneman, B., Ulmen, J., Stuart, H., Cutkosky, M. R., Kim, S., et al. (2014). Design and testing of a selectively compliant underactuated hand. Int. J. Robot. Res. 33, 721-735. doi:10.1177/0278364913518997

Aversi-Ferreira, R. A., Maior, R. S., Aziz, A., Ziermann, J. M., Nishijo, H., Tomaz, C., et al. (2014). Anatomical analysis of thumb opponency movement in the capuchin monkey (Sapajus sp). PLoS ONE 9:e87288. doi:10.1371/journal. pone. 0087288

Battezzato, A. (2015). Kinetostatic analysis and design optimization of an $\mathrm{n}$-finger underactuated hand exoskeleton. Mech. Mach. Theory 88, 86-104. doi:10.1016/j. mechmachtheory.2014.12.007

Bekey, G. A., Tomovic, R., and Zeljkovic, I. (1990). "Control architecture for the Belgrade/USC hand," in Dextrous Robot Hands, eds S. T. Venkataraman, and T. Iberall (New York, NY: Springer), 136-149.

Belter, J. T., and Dollar, A. M. (2011). "Performance characteristics of anthropomorphic prosthetic hands," in IEEE International Conference on Rehabilitation Robotics (ICORR), Vol. 29 (Zurich: CiteseerX), 1-7.

Belter, J. T., and Dollar, A. M. (2013). "Novel differential mechanism enabling two DOF from a single actuator: application to a prosthetic hand," in IEEE International Conference on Rehabilitation Robotics (ICORR), Seattle, WA, 1-6.

Belter, J. T., Segil, J. L., Dollar, A. M., and Weir, R. F. (2013). Mechanical design and performance specifications of anthropomorphic prosthetic hands: a review. J. Rehabil. Res. Dev. 50, 599. doi:10.1682/JRRD.2011.10.0188

Benefit, B. R., and McCrossin, M. L. (1997). Earliest known old world monkey skull. Nature 388, 368-371. doi:10.1038/41078

Benjamin, M., Kaiser, E., and Milz, S. (2008). Structure-function relationships in tendons: a review. J. Anat. 212, 211-228. doi:10.1111/j.1469-7580.2008.00864.x

Biagiotti, L., Lotti, F., Melchiorri, C., and Vassura, G. (2003). "Mechatronic design of innovative fingers for anthropomorphic robot hands," in IEEE International Conference on Robotics and Automation (ICRA), Vol. 3 (Taipei), 3187-3192.

Biagiotti, L., Lotti, F., Melchiorri, C., and Vassura, G. (2004). How Far is the Human Hand? A Review on Anthropomorphic Robotic End-Effectors. Bologna: University of Bologna, Tech. Rep.

Bianchi, M., Salaris, P., and Bicchi, A. (2013). Synergy-based hand pose sensing: optimal glove design. Int. J. Robot. Res. 32, 407-424. doi:10.1177/0278364912474078

Bicchi, A. (2000). Hands for dexterous manipulation and robust grasping: a difficult road toward simplicity. IEEE Trans. Robot. Autom. 16, 652-662. doi:10.1109/70.897777

Bicchi, A., Gabiccini, M., and Santello, M. (2011). Modelling natural and artificial hands with synergies. Phil. Trans. R. Soc. B 366, 3153-3161. doi:10.1098/ rstb.2011.0152

Bitzer, S., and Van Der Smagt, P. (2006). "Learning EMG control of a robotic hand: towards active prostheses," in IEEE International Conference on Robotics and Automation (ICRA), Orlando, FL, 2819-2823.

Bonilla, M., Farnioli, E., Piazza, C., Catalano, M., Grioli, G., Garabini, M., et al. (2014). "Grasping with soft hands," in IEEE-RAS International Conference on Humanoid Robots, Madrid, 581-587.

Bridgwater, L. B., Ihrke, C., Diftler, M. A., Abdallah, M. E., Radford, N. A., Rogers, J., et al. (2012). "The robonaut 2 hand-designed to do work with tools," in IEEE International Conference on Robotics and Automation (ICRA), St. Paul, MN, $3425-3430$.

Brown, C. Y., and Asada, H. H. (2007). "Inter-finger coordination and postural synergies in robot hands via mechanical implementation of principal components analysis," in IEEE/RSJ International Conference on Intelligent Robots and Systems (IROS), San Diego, CA, 2877-2882.

Buchholz, B., and Armstrong, T. J. (1992). A kinematic model of the human hand to evaluate its prehensile capabilities. J. Biomech. 25, 149-162. doi:10.1016/0021-9290(92)90272-3

Bullock, I. M., Borràs, J., and Dollar, A. M. (2012). "Assessing assumptions in kinematic hand models: a review," in 4th IEEE RAS \& EMBS International Conference on Biomedical Robotics and Biomechatronics (BioRob), Rome, 139-146.

Bullock, I. M., Zheng, J. Z., De La Rosa, S., Guertler, C., and Dollar, A. M. (2013). Grasp frequency and usage in daily household and machine shop tasks. IEEE Trans. Haptics 6, 296-308. doi:10.1109/TOH.2013.6

Butterfaß, J., Grebenstein, M., Liu, H., and Hirzinger, G. (2001). "DLR-Hand II: next generation of a dextrous robot hand," in IEEE International Conference on Robotics and Automation (ICRA), Vol. 1 (Seoul), 109-114.
Carrozza, M. C., Cappiello, G., Micera, S., Edin, B. B., Beccai, L., and Cipriani, C. (2006). Design of a cybernetic hand for perception and action. Biol. Cybern. 95, 629-644. doi:10.1007/s00422-006-0124-2

Carrozza, M. C., Suppo, C., Sebastiani, F., Massa, B., Vecchi, F., Lazzarini, R., et al. (2004). The SPRING hand: development of a self-adaptive prosthesis for restoring natural grasping. Auton. Robots 16, 125-141. doi:10.1023/ B:AURO.0000016863.48502.98

Catalano, M. G., Grioli, G., Farnioli, E., Serio, A., Piazza, C., and Bicchi, A. (2014). Adaptive synergies for the design and control of the Pisa/IIT softhand. Int. J. Robot. Res. 33, 768-782. doi:10.1177/0278364913518998

Cempini, M., Cortese, M., and Vitiello, N. (2015). A powered finger-thumb wearable hand exoskeleton with self-aligning joint axes. IEEE/ASME Trans. Mechatron. 20, 705-716. doi:10.1109/TMECH.2014.2315528

Cerruti, G., Chablat, D., Gouaillier, D., and Sakka, S. (2015). "Design method for an anthropomorphic hand able to gesture and grasp," in IEEE International Conference on Robotics and Automation (ICRA), Seattle, WA, 3660-3667.

Chalon, M., Grebenstein, M., Wimböck, T., and Hirzinger, G. (2010). “The thumb: guidelines for a robotic design," in IEEE/RSJ International Conference on Intelligent Robots and Systems (IROS), Taipei, 5886-5893.

Chang, L. Y., and Matsuoka, Y. (2006). "A kinematic thumb model for the ACT hand," in Proceedings IEEE International Conference on Robotics and Automation (ICRA) (Orlando, FL: IEEE), 1000-1005.

Chang, L. Y., and Pollard, N. S. (2008). Method for determining kinematic parameters of the in vivo thumb carpometacarpal joint. IEEE Trans. Biomed. Eng. 55, 1897-1906. doi:10.1109/TBME.2008.919854

Ciocarlie, M., and Allen, P. (2010). "Data-driven optimization for underactuated robotic hands," in IEEE International Conference on Robotics and Automation (ICRA), Anchorage, AK, 1292-1299.

Ciocarlie, M., Goldfeder, C., and Allen, P. (2007). "Dimensionality reduction for hand-independent dexterous robotic grasping," in IEEE/RSJ International Conference on Intelligent Robots and Systems (IROS), San Diego, CA, 3270-3275.

Ciocarlie, M. T., and Allen, P. K. (2009). Hand posture subspaces for dexterous robotic grasping. Int. J. Robot. Res. 28, 851-867. doi:10.1177/0278364909105606

Cipriani, C., Zaccone, F., Micera, S., and Carrozza, M. C. (2008). On the shared control of an EMG-controlled prosthetic hand: analysis of user-prosthesis interaction. IEEE Trans. Robot. 24, 170-184. doi:10.1109/TRO.2007.910708

Coert, J., van Dijke, G., Hovius, S., Snijders, C., and Meek, M. (2003). Quantifying thumb rotation during circumduction utilizing a video technique. J. Orthop. Res. 21, 1151-1155. doi:10.1016/S0736-0266(03)00114-1

Colditz, J. C. (2000). The biomechanics of a thumb carpometacarpal immobilization splint: design and fitting. J. Hand Ther. 13, 228-235. doi:10.1016/ S0894-1130(00)80006-X

Cooney, W. P., Linscheid, R. L., and An, K. N. (1984). Opposition of the thumb: an anatomic and biomechanical study of tendon transfers. J. Hand Surg. Am. 9, 777-786. doi:10.1016/S0363-5023(84)80048-9

Cooney, W. P., Lucca, M. J., Chao, E., and Linscheid, R. (1981). The kinesiology of the thumb trapeziometacarpal joint. J. Bone Joint Surg. 63, 1371-1381. doi:10.2106/00004623-198163090-00002

Cotugno, G., Althoefer, K., and Nanayakkara, T. (2016). The role of the thumb: study of finger motion in grasping and reachability space in human and robotic hands. IEEE Trans. Syst. Man, Cybern. B 47, 1061-1070. doi:10.1109/ TSMC.2016.2531679

Cotugno, G., Mohan, V., Althoefer, K., and Nanayakkara, T. (2014). "Simplifying grasping complexity through generalization of kinaesthetically learned synergies," in IEEE International Conference on Robotics and Automation (ICRA), Hong Kong, 5345-5351.

Cui, L., Cupcic, U., and Dai, J. S. (2011). "Kinematic mapping and calibration of the thumb motions for teleoperating a humanoid robot hand," in ASME 2011 International Design Engineering Technical Conferences and Computers and Information in Engineering Conference (Washington, DC: American Society of Mechanical Engineers), 1139-1147.

Cutkosky, M. R., and Howe, R. D. (1990). "Human grasp choice and robotic grasp analysis," in Dextrous Robot Hands, eds S. T. Venkataraman, and T. Iberall (New York, NY: Springer), 5-31.

Dai, J. (2005). Robotic Hand With Palm Section Comprising Several Parts Able to Move Relative to Each Other. US Patent App. 11/587,766.

Dai, J. S., and Jones, J. R. (1999). Mobility in metamorphic mechanisms of foldable/ erectable kinds. J. Mech. Des. 121, 375-382. doi:10.1115/1.2829470 
Dai, J. S., Wang, D., and Cui, L. (2009). Orientation and workspace analysis of the multifingered metamorphic hand-metahand. IEEE Trans. Robot. 25, 942-947. doi:10.1109/TRO.2009.2017138

Dechev, N., Cleghorn, W., and Naumann, S. (2001). Multiple finger, passive adaptive grasp prosthetic hand. Mech. Mach. Theory 36, 1157-1173. doi:10.1016/ S0094-114X(01)00035-0

Deimel, R., and Brock, O. (2016). A novel type of compliant and underactuated robotic hand for dexterous grasping. Int. J. Robot. Res. 35, 161-185. doi:10.1177/0278364915592961

Dermitzakis, K., Ioannides, A., and Lin, H. T. (2013). "Robotic thumb grasp-based range of motion optimisation," in 35th Annual International Conference of the IEEE Engineering in Medicine and Biology Society (EMBC), Osaka, 3163-3166.

Deshpande, A. D., Xu, Z., Weghe, M. J. V., Brown, B. H., Ko, J., Chang, L. Y., et al. (2013). Mechanisms of the anatomically correct testbed hand. IEEE/ASME Trans. Mechatron. 18, 238-250. doi:10.1109/TMECH.2011.2166801

Diftler, M., Ihrke, C., Bridgwater, L., Davis, D., Linn, D., Laske, E., et al. (2014). "Roboglove - a robonaut derived multipurpose assistive device," in IEEE International Conference on Robotics and Automation (ICRA), Hong Kong.

Diftler, M. A., Mehling, J., Abdallah, M. E., Radford, N. A., Bridgwater, L. B., Sanders, A. M., et al. (2011). "Robonaut 2-the first humanoid robot in space," in IEEE International Conference on Robotics and Automation (ICRA), Shanghai, 2178-2183.

Dollar, A. M., and Howe, R. D. (2010). The highly adaptive SDM hand: design and performance evaluation. Int. J. Robot. Res. 29, 585-597. doi:10.1177/ 0278364909360852

ElKoura, G., and Singh, K. (2003). "Handrix: animating the human hand," in Proceedings of the 2003 ACM SIGGRAPH/Eurographics Symposium on Computer Animation (San Diego, CA: Eurographics Association), 110-119.

Emerson, E. T., Krizek, T. J., and Greenwald, D. P. (1996). Anatomy, physiology, and functional restoration of the thumb. Ann. Plast. Surg. 36, 180-191. doi:10.1097/00000637-199602000-00014

Feix, T., Pawlik, R., Schmiedmayer, H.-B., Romero, J., and Kragic, D. (2009). "A comprehensive grasp taxonomy," in Robotics, Science and Systems: Workshop on Understanding the Human Hand for Advancing Robotic Manipulation, Seattle, WA, 2-3.

Feix, T., Romero, J., Schmiedmayer, H.-B., Dollar, A. M., and Kragic, D. (2016). The GRASP taxonomy of human grasp types. IEEE Trans. Human-Mach. Syst. 46, 66-77. doi:10.1109/THMS.2015.2470657

Flatt, A. E. (2002). "Our thumbs," in Baylor University Medical Center Proceedings, Vol. 15 (Dallas, TX: The Baylor University Medical Center), 380-388.

Folgheraiter, M., and Gini, G. (2000). "Blackfingers: an artificial hand that copies human hand in structure, size, and functions," in In First IEEE-RAS Int. Conf. on Humanoid Robots (Cambridge, MA: Citeseer).

Franchi, G., and Hauser, K. (2014). Technical Report: Use of Hybrid Systems to Model the Robotiq Adaptive Gripper, Bloomington, IN.

Fukaya, N., Toyama, S., Asfour, T., and Dillmann, R. (2000). "Design of the TUAT/ Karlsruhe humanoid hand," in IEEE/RSJ International Conference on Intelligent Robots and Systems (IROS), Vol. 3 (Takamatsu), 1754-1759.

Gabiccini, M., Bicchi, A., Prattichizzo, D., and Malvezzi, M. (2011). On the role of hand synergies in the optimal choice of grasping forces. Auton. Robots 31, 235-252. doi:10.1007/s10514-011-9244-1

Gaiser, I. N., Pylatiuk, C., Schulz, S., Kargov, A., Oberle, R., and Werner, T. (2009). The FLUIDHAND III: a multifunctional prosthetic hand. J. Prosthet. Orthot. 21, 91-96. doi:10.1097/JPO.0b013e3181a1ca54

Gama Melo, E. N., Aviles Sanchez, O. F., and Amaya Hurtado, D. (2014). Anthropomorphic robotic hands: a review. Ingeniería y Desarrollo 32, 279-313. doi:10.14482/inde.32.2.4715

Giurintano, D., Hollister, A., Buford, W., Thompson, D., and Myers, L. (1995). A virtual five-link model of the thumb. Med. Eng. Phys. 17, 297-303. doi:10.1016/1350-4533(95)90855-6

Gosselin, C., Pelletier, F., and Laliberte, T. (2008). "An anthropomorphic underactuated robotic hand with 15 DOFs and a single actuator," in IEEE International Conference on Robotics and Automation (ICRA), Pasadena, CA, 749-754.

Gray, H. (1918). Anatomy of the Human Body. Philadelphia, PA: Lea \& Febiger.

Grebenstein, M. (2012). Approaching Human Performance. Ph.D. thesis, Springer, Zurich.

Grebenstein, M., Chalon, M., Herzinger, G., and Siegwart, R. (2010). "A method for hand kinematics designers: 7 billion perfect hands," in 1st International Conference on Applied Bionics and Biomechanics (ICABB), Venice.
Greene, D. P., and Roberts, S. L. (2015). Kinesiology: Movement in the Context of Activity. St. Louis, MO: Elsevier Health Sciences.

Greenhill, R., Ellas, H., Walker, R., and Godden, M. (2010). End Effectors. US Patent $7,673,916$.

Greiner, T. M. (1991). Hand Anthropometry of US Army Personnel. Technical Report, Natick, MA: DTIC Document.

Griffin, W. B., Findley, R. P., Turner, M. L., and Cutkosky, M. R. (2000). “Calibration and mapping of a human hand for dexterous telemanipulation," in ASME IMECE 2000 Symposium on Haptic Interfaces for Virtual Environments and Teleoperator Systems, Orlando, FL, 1-8.

Grinyagin, I. V., Biryukova, E. V., and Maier, M. A. (2005). Kinematic and dynamic synergies of human precision-grip movements. J. Neurophysiol. 94, 2284-2294. doi: $10.1152 /$ jn.01310.2004

Hart, D. L., Isernhagen, S. J., and Matheson, L. N. (1993). Guidelines for functional capacity evaluation of people with medical conditions 1. J. Orthop. Sports Phys. Ther. 18, 682-686. doi:10.2519/jospt.1993.18.6.682

Hasegawa, Y., Ariyama, T., and Kamibayashi, K. (2012). "Pinching force accuracy affected by thumb sensation in human force augmentation," in IEEE/RSJ International Conference on Intelligent Robots and Systems (IROS), Vilamoura, 3943-3948.

Haslam, M., Hernandez-Aguilar, A., Ling, V., Carvalho, S., de La Torre, I., DeStefano, A., et al. (2009). Primate archaeology. Nature 460, 339-344. doi: $10.1038 /$ nature 08188

Heo, P., Gu, G. M., Lee, S.-J., Rhee, K., and Kim, J. (2012). Current hand exoskeleton technologies for rehabilitation and assistive engineering. Int. J. Precis. Eng. Manuf. 13, 807-824. doi:10.1007/s12541-012-0107-2

Hoffmann, M., and Pfeifer, R. (2012). "The implications of embodiment for behavior and cognition: animal and robotic case studies," in The Implications of Embodiment: Cognition and Communications, eds W. Tschacher, and C. Bergomi (Imprint Academic), 31-58.

Hollister, A., Buford, W., Myers, L., Giurintano, D., and Novick, A. (1992). The axes of rotation of the thumb carpometacarpal joint. J. Orthop. Res. 10, 454-460. doi:10.1002/jor.1100100319

Hollister, A., Giurintano, D. J., Buford, W. L., Myers, L. M., and Novick, A. (1995). The axes of rotation of the thumb interphalangeal and metacarpophalangeal joints. Clin. Orthop. Relat. Res. 320, 188-193.

Hoshino, K., and Kawabuchi, I. (2005). "Pinching with finger tips in humanoid robot hand," in 12th International Conference on Advanced Robotics (ICAR), Seattle, WA, 705-712.

Hume, M. C., Gellman, H., McKellop, H., and Brumfield, R. H. (1990). Functional range of motion of the joints of the hand. J. Hand Surg. Am. 15, 240-243. doi:10.1016/0363-5023(90)90102-W

Iberall, T. (1987). "The nature of human prehension: three dextrous hands in one," in IEEE International Conference on Robotics and Automation (ICRA), Vol. 4 (Raleigh, NC), 396-401.

Imaeda, T., Niebur, G., Cooney, W. P., Linscheid, R. L., and An, K.-N. (1994). Kinematics of the normal trapeziometacarpal joint. J. Orthop. Res. 12, 197-204. doi:10.1002/jor.1100120209

Ingram, J. N., Körding, K. P., Howard, I. S., and Wolpert, D. M. (2008). The statistics of natural hand movements. Exp. Brain Res. 188, 223-236. doi:10.1007/ s00221-008-1355-3

Inouye, J. M., and Valero-Cuevas, F. J. (2013). Anthropomorphic tendon-driven robotic hands can exceed human grasping capabilities following optimization. Int. J. Robot. Res. 33, 694-705. doi:10.1177/0278364913504247

Iwata, H., and Sugano, S. (2009). "Design of human symbiotic robot TWENDYONE," in IEEE International Conference on Robotics and Automation (ICRA), Kobe, 580-586.

Jacobsen, S., Iversen, E., Knutti, D., Johnson, R., and Biggers, K. (1986). “Design of the Utah/MIT dextrous hand," in IEEE International Conference on Robotics and Automation (ICRA), Vol. 3 (San Francisco, CA), 1520-1532.

Jeannerod, M. (1984). The timing of natural prehension movements. J. Mot. Behav. 16, 235-254. doi:10.1080/00222895.1984.10735319

Jones, L. A., and Lederman, S. J. (2006). Human Hand Function. New York, NY: Oxford University Press.

Kandel, E. R., Schwartz, J. H., and Jessell, T. M. (2000). Principles of Neural Science, Vol. 4. New York: McGraw-Hill.

Kang, B. B., Lee, H., In, H., Jeong, U., Chung, J., and Cho, K.-J. (2016). “Development of a polymer-based tendon-driven wearable robotic hand," in IEEE International Conference on Robotics and Automation (ICRA), Stockholm, 3750-3755. 
Kapandji, I. (1982). "The physiology of the joints: upper limb," in Physiology of the Joints, ed. L. H. Honoré (Edinburgh: Churchill Livingstone) [translated by L. H. Honoré; preface by F. Poilleux].

Kargov, A., Ivlev, O., Pylatiuk, C., Asfour, T., Schulz, S., Gräser, A., et al. (2007). Applications of a Fluidic Artificial Hand in the Field of Rehabilitation. Vienna: Rehabilitation Robotics.

Kargov, A., Pylatiuk, C., Martin, J., Schulz, S., and Döderlein, L. (2004). A comparison of the grip force distribution in natural hands and in prosthetic hands. Disabil. Rehabil. 26, 705-711. doi:10.1080/09638280410001704278

Kaufman, K. R., An, K.-N., Litchy, W. J., Cooney, W. P., and Chao, E. Y. (1999). In-vivo function of the thumb muscles. Clin. Biomech. 14, 141-150. doi:10.1016/ S0268-0033(98)00058-8

Kawasaki, H., Komatsu, T., and Uchiyama, K. (2002). Dexterous anthropomorphic robot hand with distributed tactile sensor: Gifu hand II. IEEE/ASME Trans. Mechatron. 7, 296-303. doi:10.1109/TMECH.2002.802720

Kilbreath, S., and Gandevia, S. (1993). Neural and biomechanical specializations of human thumb muscles revealed by matching weights and grasping objects. J. Physiol. 472, 537-556. doi:10.1113/jphysiol.1993.sp019961

Kivell, T. (2015). Evidence in hand: recent discoveries and the early evolution of human manual manipulation. Philos. Trans. R. Soc. Lond. B Biol. Sci. 370, 1-11. doi:10.1098/rstb.2015.0105

Konnaris, C., Gavriel, C., Thomik, A. A., and Faisal, A. A. (2016). "Ethohand: a dexterous robotic hand with ball-joint thumb enables complex in-hand object manipulation," in 6th IEEE International Conference on Biomedical Robotics and Biomechatronics (BioRob), Singapore, 1154-1159.

Kuczynski, K. (1974). Carpometacarpal joint of the human thumb. J. Anat. 118, 119.

Lalibert, T., Baril, M., Guay, F., and Gosselin, C. (2010). "Towards the design of a prosthetic underactuated hand," in Proc. IFToMM/ASME Int. Workshop Underactuated Grasping, Montreal, 19-26.

Lane, D. M., Davies, J. B. C., Robinson, G., O’Brien, D. J., Sneddon, J., Seaton, E., et al. (1999). The AMADEUS dextrous subsea hand: design, modeling, and sensor processing. IEEE J. Oceanic Eng. 24, 96-111. doi:10.1109/48. 740158

Laschi, C., Cianchetti, M., Mazzolai, B., Margheri, L., Follador, M., and Dario, P. (2012). Soft robot arm inspired by the octopus. Adv. Robot. 26, 709-727. doi: $10.1163 / 156855312 X 626343$

Lewis, O. J. (1977). Joint remodelling and the evolution of the human hand. J. Anat. $123,157-201$

Li, Z.-M., and Tang, J. (2007). Coordination of thumb joints during opposition. J. Biomech. 40, 502-510. doi:10.1016/j.jbiomech.2006.02.019

Liarokapis, M. V., Artemiadis, P. K., and Kyriakopoulos, K. J. (2013). "Quantifying anthropomorphism of robot hands," in IEEE International Conference on Robotics and Automation (ICRA), Karlsruhe, 2041-2046.

Light, C., and Chappell, P. (2000). Development of a lightweight and adaptable multiple-axis hand prosthesis. Med. Eng. Phys. 22, 679-684. doi:10.1016/ S1350-4533(01)00017-0

Lin, T. W., Cardenas, L., and Soslowsky, L. J. (2004). Biomechanics of tendon injury and repair. J. Biomech. 37, 865-877. doi:10.1016/j.jbiomech.2003.11.005

Liu, H., Butterfass, J., Knoch, S., Meusel, P., and Hirzinger, G. (1999). A new control strategy for DLR's multisensory articulated hand. IEEE Control Syst. 19, 47-54. doi:10.1109/37.753935

Liu, H., Meusel, P., Seitz, N., Willberg, B., Hirzinger, G., Jin, M., et al. (2007). The modular multisensory DLR-HIT-Hand. Mech. Mach. Theory 42, 612-625. doi:10.1016/j.mechmachtheory.2006.04.013

Liu, H., Wu, K., Meusel, P., Seitz, N., Hirzinger, G., Jin, M., et al. (2008). "Multisensory five-finger dexterous hand: the DLR/HIT Hand II," in IEEE/RSJ International Conference on Intelligent Robots and Systems (IROS), Nice, 3692-3697.

Lotti, F., Tiezzi, P., Vassura, G., Biagiotti, L., and Melchiorri, C. (2004). "UBH 3: an anthropomorphic hand with simplified endo-skeletal structure and soft continuous fingerpads," in IEEE International Conference on Robotics and Automation (ICRA), Vol. 5 (New Orleans, LA), 4736-4741.

Loucks, C., Johnson, V., Boissiere, P., Starr, G., and Steele, J. (1987). “Modeling and control of the Stanford/JPL hand," in IEEE International Conference on Robotics and Automation (ICRA), Vol. 4 (Raleigh, NC), 573-578.

Lovchik, C., and Diftler, M. A. (1999). "The robonaut hand: a dexterous robot hand for space," in IEEE International Conference on Robotics and Automation (ICRA), Vol. 2 (Detroit, MI), 907-912.

Ma, R. R., and Dollar, A. M. (2011). "On dexterity and dexterous manipulation," in 15th International Conference on Advanced Robotics (ICAR), Tallinn, 1-7.
Ma, R. R., Odhner, L. U., and Dollar, A. M. (2013). "A modular, open-source 3D printed underactuated hand," in IEEE International Conference on Robotics and Automation (ICRA), Karlsruhe, 2737-2743.

Martell, J. S., and Gini, G. (2007). "Robotic hands: design review and proposal of new design process," Proceedings of World Academy of Science, Engineering and Technology (WASET) [Int. J. Mech. Aerosp. Indus. Mech. Manuf. Eng. 1(2), 57-62].

Marzke, M. W. (1997). Precision grips, hand morphology, and tools. Am. J. Phys. Anthropol. 102, 91-110. doi:10.1002/(SICI)1096-8644(199701)1021<91::AIDAJPA8 $>3.0 . \mathrm{CO} ; 2-\mathrm{G}$

Marzke, M. W. (2013). Tool making, hand morphology and fossil hominins. Philos. Trans. R. Soc. B Biol. Sci. 368, 20120414. doi:10.1098/rstb.2012.0414

Marzke, M. W., and Marzke, R. F. (2000). Evolution of the human hand: approaches to acquiring, analysing and interpreting the anatomical evidence. J. Anat. 197, 121-140. doi:10.1046/j.1469-7580.2000.19710121.x

Massa, B., Roccella, S., Carrozza, M. C., and Dario, P. (2002). "Design and development of an underactuated prosthetic hand," in IEEE International Conference on Robotics and Automation (ICRA), Vol. 4 (Washington, DC), 3374-3379.

McFarlane, R. (1962). Observations on the functional anatomy of the intrinsic muscles of the thumb. J. Bone Joint Surg. Am. 44, 1073-1088. doi:10.2106/ 00004623-196244060-00004

Medynski, C., and Rattray, B. (2011). "Bebionic prosthetic design," in Myoelectric Symposium, New Brunswick.

Miyata, N., Kouchi, M., Kurihara, T., and Mochimaru, M. (2004). "Modeling of human hand link structure from optical motion capture data," in IEEE/ RSJ International Conference on Intelligent Robots and Systems (IROS), Vol. 3 (Sendai), 2129-2135.

Mouri, T., Kawasaki, H., Yoshikawa, K., Takai,J., and Ito,S. (2002). "Anthropomorphic robot hand: Gifu hand III," in International Conference on Control, Automation and Systems (ICCAS), Jeonbuk, 1288-1293.

Nanayakkara, V., Ataka, A., Venetsanos, D., Duran, O., Vitzilaios, N., Nanayakkara, T., et al. (2016). "Kinematic analysis of the human thumb with foldable palm," in Conference towards Autonomous Robotic Systems (TAROS), eds L. Alboul D. Damian, and J. M. Aitken (Sheffield: Springer), 226-238.

Napier, J. R. (1956). The prehensile movements of the human hand. J. Bone Joint Surg. 38, 902-913.

Neumann, D. A., and Bielefeld, T. (2003). The carpometacarpal joint of the thumb: stability, deformity, and therapeutic intervention. J. Orthop. Sports Phys. Ther. 33, 386-399. doi:10.2519/jospt.2003.33.7.386

Odhner, L. U., Jentoft, L. P., Claffee, M. R., Corson, N., Tenzer, Y., Ma, R. R., et al. (2014). A compliant, underactuated hand for robust manipulation. Int. J. Robot. Res. 33, 736-752. doi:10.1177/0278364913514466

Okada, T. (1982). Computer control of multijointed finger system for precise object-handling. IEEE Trans. Syst. Man Cybern. 12, 289-299. doi:10.1109/ TSMC.1982.4308818

Ozawa, R., Hashirii, K., Yoshimura, Y., Moriya, M., and Kobayashi, H. (2014). Design and control of a three-fingered tendon-driven robotic hand with active and passive tendons. Auton. Robots 36, 67-78. doi:10.1007/s10514013-9362-z

Pao, L., and Speeter, T. H. (1989). "Transformation of human hand positions for robotic hand control," in IEEE International Conference on Robotics and Automation (ICRA), Scottsdale, AZ, 1758-1763.

Parida, P. K. (2013). Kinematic Analysis of Multi-Fingered, Anthropomorphic Robotic Hands. Ph.D. thesis, National Institute of Technology Rourkela, Odisha.

Peerdeman, B., Valori, M., Brouwer, D., Hekman, E., Misra, S., and Stramigioli, S. (2014). UT hand I: a lock-based underactuated hand prosthesis. Mech. Mach. Theory 78, 307-323. doi:10.1016/j.mechmachtheory.2014.03.018

Pfeifer, R., Iida, F., and Lungarella, M. (2014). Cognition from the bottom up: on biological inspiration, body morphology, and soft materials. Trends Cogn. Sci. 18, 404-413. doi:10.1016/j.tics.2014.04.004

Polygerinos, P., Wang, Z., Galloway, K. C., Wood, R. J., and Walsh, C. J. (2015). Soft robotic glove for combined assistance and at-home rehabilitation. Rob. Auton. Syst. 73, 135-143. doi:10.1016/j.robot.2014.08.014

Pons, J., Ceres, R., and Pfeiffer, F. (1999). Multifingered dextrous robotics hand design and control: a review. Robotica 17, 661-674. doi:10.1017/S0263574799001836

Pons, J., Rocon, E., Ceres, R., Reynaerts, D., Saro, B., Levin, S., et al. (2004). The MANUS-HAND dextrous robotics upper limb prosthesis: mechanical and manipulation aspects. Auton. Robots 16, 143-163. doi:10.1023/ B:AURO.0000016862.38337.f1 
Pouydebat, E., Laurin, M., Gorce, P., and Bels, V. (2008). Evolution of grasping among anthropoids. J. Evol. Biol. 21, 1732-1743. doi:10.1111/j.1420-9101.2008.01582.x

Prattichizzo, D., Malvezzi, M., Hussain, I., and Salvietti, G. (2014). "The sixthfinger: a modular extra-finger to enhance human hand capabilities," in The 23rd IEEE International Symposium on Robot and Human Interactive Communication (RO-MAN), Edinburgh, 993-998.

Pulleyking, S., Das, D., and Schultz, J. (2016). "Simplified robotic thumb inspired by surgical intervention," in 6th IEEE International Conference on Biomedical Robotics and Biomechatronics (BioRob), Singapore, 1200-1206.

Ramos, A. M., Gravagne, I. A., and Walker, I. D. (1999). "Goldfinger: a non-anthropomorphic, dextrous robot hand," in IEEE International Conference on Robotics and Automation (ICRA), Vol. 2 (Detroit, MI), 913-919.

Richards, D. S., Georgilas, I., Dagnino, G., and Dogramadzi, S. (2015). "Powered exoskeleton with palm degrees of freedom for hand rehabilitation," in 37th Annual International Conference of the IEEE Engineering in Medicine and Biology Society (EMBC), Milan, 4635-4638.

Rolian, C., Lieberman, D. E., and Zermeno, J. P. (2011). Hand biomechanics during simulated stone tool use. J. Hum. Evol. 61, 26-41. doi:10.1016/j. jhevol.2011.01.008

Rubinger, B., Fulford, P., Gregoris, L., Gosselin, C., and Laliberté, T. (2001). "Self-adapting robotic auxiliary hand (SARAH) for SPDM operations on the international space station," in Proceedings of I-SAIRAS (Quebec, Canada).

Rus, D., and Tolley, M. T. (2015). Design, fabrication and control of soft robots. Nature 521, 467-475. doi:10.1038/nature14543

Sangole, A. P., and Levin, M. F. (2008). Arches of the hand in reach to grasp. J. Biomech. 41, 829-837. doi:10.1016/j.jbiomech.2007.11.006

Santello, M., Baud-Bovy, G., and Jörntell, H. (2013). Neural bases of hand synergies. Front. Comput. Neurosci. 7:23. doi:10.3389/fncom.2013.00023

Santello, M., Bianchi, M., Gabiccini, M., Ricciardi, E., Salvietti, G., Prattichizzo, D., et al. (2016). Hand synergies: integration of robotics and neuroscience for understanding the control of biological and artificial hands. Phys. Life Rev. 17, 1-23. doi:10.1016/j.plrev.2016.02.001

Santello, M., Flanders, M., and Soechting, J. F. (1998). Postural hand synergies for tool use. J. Neurosci. 18, 10105-10115.

Schieber, M. H. (1995). Muscular production of individuated finger movements: the roles of extrinsic finger muscles. J. Neurosci. 15, 284-297.

Schmitz, A., Pattacini, U., Nori, F., Natale, L., Metta, G., and Sandini, G. (2010). "Design, realization and sensorization of the dexterous icub hand," in 10th IEEE-RAS International Conference on Humanoid Robots, Nashville, TN, 186-191.

Schulz, S., Pylatiuk, C., and Bretthauer, G. (2001). "A new ultralight anthropomorphic hand," in IEEE International Conference on Robotics and Automation (ICRA), Vol. 3 (Seoul), 2437-2441.

ShadowHand. (2013). Shadow Dexterous Hand, E1 Series (E1M3R, E1M3L, E1P1R, E1P1L) Technical Specification (London: Shadow Robot Company).

Shimizu, S., Shimojo, M., Sato, S., Seki, Y., Takahashi, A., Inukai, Y., et al. (1997). "The relationship between human grip types and force distribution pattern in grasping," in 8th International Conference on Advanced Robotics (ICAR), Monterey, CA, 299-304.

Smutz, W. P., Kongsayreepong, A., Hughes, R. E., Niebur, G., Cooney, W. P., and An, K.-N. (1998). Mechanical advantage of the thumb muscles. J. Biomech. 31, 565-570. doi:10.1016/S0021-9290(98)00043-8

Sornkarn, N., Dasgupta, P., and Nanayakkara, T. (2016). Morphological computation of haptic perception of a controllable stiffness probe. PLOS ONE 11:e0156982. doi:10.1371/journal.pone.0156982

Sornkarn, N., Howard, M., and Nanayakkara, T. (2014). "Internal impedance control helps information gain in embodied perception," in IEEE International Conference on Robotics and Automation (ICRA), Hong Kong, 6685-6690.

Stillfried, G., Hillenbrand, U., Settles, M., and van der Smagt, P. (2014). "MRI-based skeletal hand movement model," in The Human Hand as an Inspiration for Robot Hand Development, eds R. Balasubramanian, and V. J. Santos (Switzerland: Springer International Publishing), 49-75.

Stillfried, G., and van der Smagt, P. (2010). "Movement model of a human hand based on magnetic resonance imaging (MRI)," in International Conference on Applied Biometarials and Biomechanics (ICABB), Venice.
Tagliabue, M., Ciancio, A. L., Brochier, T., Eskiizmirliler, S., and Maier, M. A. (2015). Differences between kinematic synergies and muscle synergies during two-digit grasping. Front. Hum. Neurosci. 9:165. doi:10.3389/fnhum.2015.00165

Tavakoli, M., Enes, B., Santos, J., Marques, L., and de Almeida, A. T. (2015). Underactuated anthropomorphic hands: actuation strategies for a better functionality. Rob. Auton. Syst. 74, 267-282. doi:10.1016/j.robot.2015.08.011

Taylor, C. L., and Schwarz, R. J. (1955). The anatomy and mechanics of the human hand. Artif. Limbs 2, 22-35.

Tomovic, R., and Boni, G. (1962). An adaptive artificial hand. IRE Trans. Autom. Control 7, 3-10. doi:10.1109/TAC.1962.1105456

Troccaz, J., and Connolly, C. (2008). Prosthetic hands from touch bionics. Ind. Robot. 35, 290-293. doi:10.1108/01439910810876364

Ueki, S., Kawasaki, H., Ito, S., Nishimoto, Y., Abe, M., Aoki, T., et al. (2012). Development of a hand-assist robot with multi-DOFs for rehabilitation therapy. IEEE/ASME Trans. Mechatron. 17, 136-146. doi:10.1109/TMECH.2010.2090353

Valero-Cuevas, F. J., Hoffmann, H., Kurse, M. U., Kutch, J. J., and Theodorou, E. A. (2009). Computational models for neuromuscular function. IEEE Rev. Biomed. Eng. 2, 110-135. doi:10.1109/RBME.2009.2034981

Valero-Cuevas, F. J., Johanson, M. E., and Towles, J. D. (2003). Towards a realistic biomechanical model of the thumb: the choice of kinematic description may be more critical than the solution method or the variability/uncertainty of musculoskeletal parameters. J. Biomech. 36, 1019-1030. doi:10.1016/ S0021-9290(03)00061-7

van der Hulst, F. P., Schätzle, S., Preusche, C., and Schiele, A. (2012). "A functional anatomy based kinematic human hand model with simple size adaptation," in IEEE International Conference on Robotics and Automation (ICRA), St. Paul, MN, 5123-5129.

von Schroeder, H. P., and Botte, M. J. (2001). Anatomy and functional significance of the long extensors to the fingers and thumb. Clin. Orthop. Relat. Res. 383, 74-83. doi:10.1097/00003086-200102000-00010

Wei, G., Dai, J. S., Wang, S., and Luo, H. (2011). Kinematic analysis and prototype of a metamorphic anthropomorphic hand with a reconfigurable palm. Int. J. Human. Robot. 8, 459-479. doi:10.1142/S0219843611002538

Wohlman, S. J., and Murray, W. M. (2013). Bridging the gap between cadaveric and in vivo experiments: a biomechanical model evaluating thumb-tip endpoint forces. J. Biomech. 46, 1014-1020. doi:10.1016/j.jbiomech.2012.10.044

Xu, Z., and Todorov, E. (2016). "Design of a highly biomimetic anthropomorphic robotic hand towards artificial limb regeneration," in IEEE International Conference on Robotics and Automation (ICRA), Stockholm, 3485-3492.

Yang, D., Zhao, J., Gu, Y., Jiang, L., and Liu, H. (2009). "EMG pattern recognition and grasping force estimation: improvement to the myocontrol of multi-DOF prosthetic hands," in IEEE/RSJ International Conference on Intelligent Robots and Systems (IROS), St. Louis, MO, 516-521.

Yang, J., Pitarch, E. P., Abdel-Malek, K., Patrick, A., and Lindkvist, L. (2004). A multi-fingered hand prosthesis. Mech. Mach. Theory 39, 555-581. doi:10.1016/ j.mechmachtheory.2004.01.002

Young, R. W. (2003). Evolution of the human hand: the role of throwing and clubbing. J. Anat. 202, 165-174. doi:10.1046/j.1469-7580.2003.00144.x

Zartl, M., Kapfer, T., and Muellbacher, W. (2014). Functional topography of cortical thumb movement representations in human primary motor cortex. Brain Topogr. 27, 228-239. doi:10.1007/s10548-013-0289-7

Zhang, X., Braido, P., Lee, S.-W., Hefner, R., and Redden, M. (2005). A normative database of thumb circumduction in vivo: center of rotation and range of motion. Hum. Factors 47, 550-561. doi:10.1518/001872005774860069

Conflict of Interest Statement: The authors declare that the research was conducted in the absence of any commercial or financial relationships that could be construed as a potential conflict of interest.

Copyright $\odot 2017$ Nanayakkara, Cotugno, Vitzilaios, Venetsanos, Nanayakkara and Sahinkaya. This is an open-access article distributed under the terms of the Creative Commons Attribution License (CC BY). The use, distribution or reproduction in other forums is permitted, provided the original author(s) or licensor are credited and that the original publication in this journal is cited, in accordance with accepted academic practice. No use, distribution or reproduction is permitted which does not comply with these terms. 